\title{
High-mass star formation toward southern infrared bubble S10
}

\author{
Swagat Ranjan Das ${ }^{1}$, Anandmayee Tej and Sarita Vig \\ Indian Institute of Space Science and Technology, Trivandrum 695547, India \\ Swarna K.Ghosh and Ishwara Chandra C.H. \\ National Centre For Radio Astrophysics, Pune 41100\%, India \\ swagat.12@iist.ac.in
}

\begin{abstract}
An investigation in radio and infrared wavelengths of two high-mass star forming regions toward the southern Galactic bubble S10 is presented here. The two regions under study are associated with the broken bubble S10 and Extended Green Object, G345.99-0.02, respectively. Radio continuum emission mapped at 610 and $1280 \mathrm{MHz}$ using the Giant Metrewave Radio Telescope, India is detected towards both the regions. These regions are estimated to be ionized by early B to late O type stars. Spitzer GLIMPSE mid-infrared data is used to identify young stellar objects associated with these regions. A Class I/II type source, with an estimated mass of $6.2 \mathrm{M}_{\odot}$, lies $\sim 7^{\prime \prime}$ from the radio peak. Pixel-wise, modified blackbody fits to the thermal dust emission using Herschel far-infrared data is performed to construct dust temperature and column density maps. Eight clumps are detected in the two regions using the $250 \mu \mathrm{m}$ image. The masses and linear diameter of these range between $\sim 300-1600 \mathrm{M}_{\odot}$ and $0.2-1.1 \mathrm{pc}$, respectively which qualifies them as high-mass star forming clumps. Modelling of the spectral energy distribution of these clumps indicates the presence of high luminosity, high accretion rate, massive young stellar objects possibly in the accelerating accretion phase. Further, based on the radio and MIR morphology, the occurrence of a possible bow-wave towards the likely ionizing star is explored.
\end{abstract}

Subject headings: stars: formation - ISM: HII region - ISM - radio continuum - ISM: individual objects (S10 - IRAS 17036-4033): individual objects (G345.99-0.02)

\section{Introduction}

High-mass stars play a crucial role in the dynamical and chemical evolution of the Galaxy considering that their feedback to the interstellar medium (ISM) is in the form of energy and heavy elements. However, these most massive members of the stellar population pose theoretical as well as observational challenges in the way of our understanding of the formation processes involved. 
For massive stars $\left(\mathrm{M} \gtrsim 8 \mathrm{M}_{\odot}\right)$, the Kelvin-Helmholtz time scale is less than the accretion time scale which implies that the star 'switches on' (reaches the main-sequence) while still accreting (McKee \& Tan 2003). This invokes the 'radiation pressure problem' that would inhibit further accretion to form a massive star. Inspite of various theories proposed to counter this problem, the decision is still not sealed on whether high-mass stars are formed via mechanisms like competitive accretion or coalescence of low-mass stars in dense protoclusters (Bonnell et al. 2004) or their formation is just a scaled up version of the processes in play in the low-mass regime which includes formation via monolithic collapse, disk accretion (with a larger accretion rate) and outflow (McKee \& Tan 2003; Yorke \& Sonnhalter 2002). Further, since high-mass stars form in clustered, highly obscured and distant ( $\sim 1 \mathrm{kpc}$ or beyond) environments, observing them is a challenging task. Hence, lack of good and adequate observational guidance has kept the theoretical models debatable. A recent review by Tan et al. 2014 discusses the current theoretical and observational scenario of high-mass star formation. Observational manifestations of the interplay between highmass stars and the surrounding ISM are important probes for studying the various evolutionary phases involved in their formation. The very early stages are marked by the presence of energetic outflows and jets. Once the 'switching-on' takes place, the outpouring of UV photons ionize the surrounding neutral medium forming HII regions (Wood \& Churchwell 1989; Churchwell 2002). The HII region around a newly formed massive star expands into the ambient ISM driven by various feedback mechanisms like thermal overpressure, powerful stellar winds, radiation pressure or a combination of all (Churchwell et al. 2006; Deharveng et al. 2010; Simpson et al. 2012). The result is a 'bubble' that shows up as a dense shell of swept up gas and dust between the ionization and the shock fronts encompassing a relatively low-density, evacuated cavity around the central star (Weaver et al. 1977). A detailed discussion on bubbles is presented in Section 4.5.

In this paper, we present an observational study of a high-mass star forming region which includes the southern Galactic bubble S10 and an Extended Green Object (EGO) G345.99-0.02 (hereafter EGO345) which is located $\sim 5^{\prime}$ toward the north-east of S10. Both these regions are shown to harbour massive protostellar candidates (Fontani et al. 2005; Beltrán et al. 2006). Figure 11 shows the mid-infrared image of the two regions studied in this paper.

The southern infrared (IR) bubble S10 is listed in Churchwell et al. (2006) as one having a broken morphology. Broken morphologies of bubbles are believed to be due to non-uniform density of the ambient ISM and/or anisotropic stellar winds and radiation fields. Based on the $24 \mu \mathrm{m}$ MIPSGAL image, these authors suggest identification of possible central driving star(s). S10 is also identified as a bubble in the Milky Way Project (Simpson et al. 2012). In Figure 1, we trace the elliptical and almost spherical morphologies of S10 as suggested by Churchwell et al. (2006) and Simpson et al. (2012), respectively. We support the larger spherical morphology of Simpson et al. (2012) given the extended southern part of the bubble. However, the thickness of $0.98^{\prime}$ estimated by them is on the higher side compared to $0.3^{\prime}$ quoted by Churchwell et al. (2006). We have adopted the latter value. A bright IRAS source (IRAS 17036-4033), with a bolometric luminosity of $2.5 \times 10^{4} \mathrm{~L}_{\odot}$ (Beltrán et al. 2006), is located towards the eastern arm of S10. The estimated 


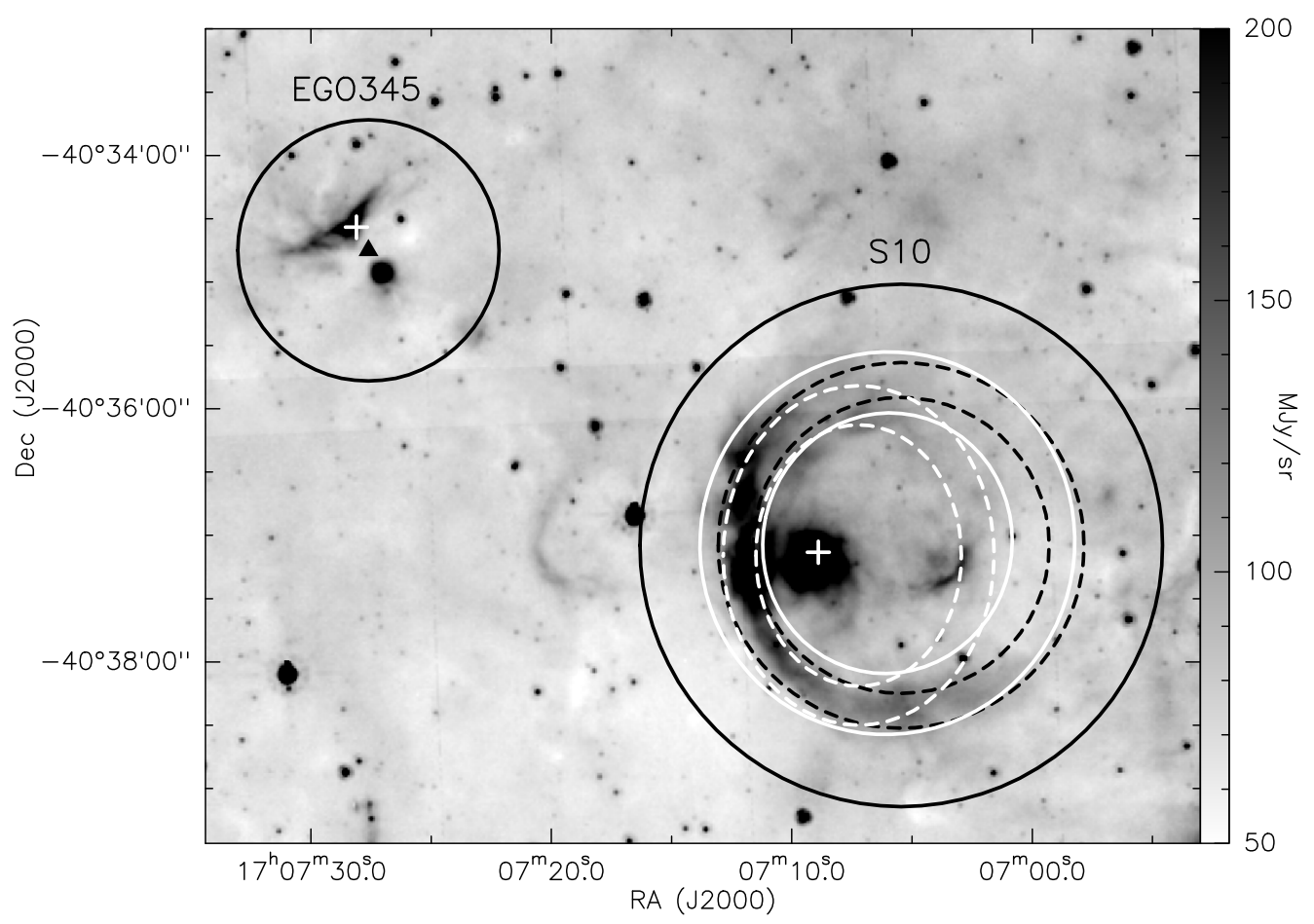

Fig. 1.- IRAC $8.0 \mu \mathrm{m}$ image of the regions (shown as black circles) probed in this paper. The '+' marks show the positions of the associated IRAS point sources, IRAS 17036-4033 (S10) and IRAS 17039-4030 (EGO345). The filled black triangle shows the location of the EGO. We show the various morphologies proposed for the bubble - white dashed (Churchwell et al. 2006); white solid (Simpson et al. 2012) and black dashed (our estimate).

centre position of S10 as given by these authors lies within the error ellipse of the IRAS point source position. An arc-type structure with an opening in the north-east direction is seen towards the west of the likely centre of the bubble.

The second region which includes EGO345 shows an extended emission to the north-east and a bright compact emission to the south-west of the EGO. The EGOs which display enhanced $4.5 \mu \mathrm{m}$ emission (given common colour coding of green in the Spitzer-GLIMPSE colour composite images and hence the name) are likely candidates tracing outflows from massive young stellar objects Cvganowski et al. 2008; Chambers et al. 2009; De Buizer \& Vacca 2010; Lee et al. 2012, 2013; Caratti o Garatti et al. 2015). In Figure 2, we display the colour composite (3.6, 4.5 and $8.0 \mu \mathrm{m}$ ) image which shows the location of the EGO. This region is associated with IRAS 170394030 (Cyganowski et al. 2008). It has no association with any Infrared Dark Clouds (IRDCs) or $\mathrm{OH}$ masers but is associated with Class I and Class II methanol masers which are signposts of high-mass star forming regions (Chen et al. 2011; Caswell et al. 2010).

Both these regions have been studied in the rotational transition lines of $\mathrm{CS}$ and $\mathrm{C}^{17} \mathrm{O}$ 


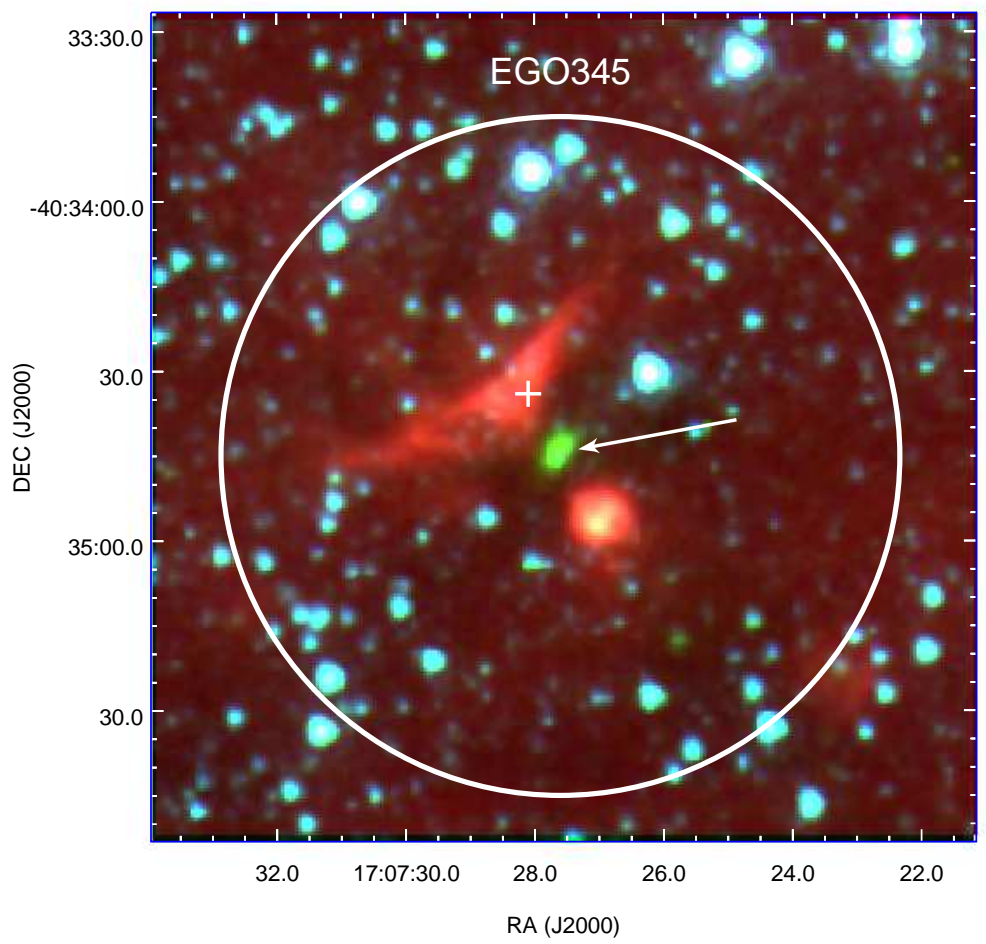

Fig. 2.- Colour composite image of EGO345 with $8.0 \mu \mathrm{m}$ (red), $4.5 \mu \mathrm{m}$ (green) and $3.6 \mu \mathrm{m}$ (blue) colour coding. The arrow points to the position of EGO345 and '+' mark shows the position of associated IRAS point source.

molecules, and $1.2 \mathrm{~mm}$ continuum emission as part of the survey for search of massive protostellar candidates using SEST telescope (Fontani et al. 2005; Beltrán et al. 2006). As discussed in Beltrán et al. (2006), the $1.2 \mathrm{~mm}$ dust continuum emission map shows the presence of six massive clumps with derived masses between $85-423 \mathrm{M}_{\odot}$. Four of these clumps are located in the eastern periphery of S10 and associated with IRAS 17036-4033. The other two clumps are towards the north-east and associated with EGO345. Beltrán et al. (2006) assume these six clumps to belong to the same star forming region. This is supported by the distance estimates to IRAS 17036-4033 and EGO345. Using the CS line velocity, Fontani et al. (2005) estimate the near and far kinematic distances for IRAS $17036-4033$ to be 5.7 and $10.8 \mathrm{kpc}$, respectively. In this paper, we adopt the near distance. The distance to the region EGO345 is also estimated to be $5.6 \mathrm{kpc}$ (Chen et al. 2011). Further, the location of the four clumps in the periphery of S10 strongly suggests a fragmented shell interacting and shaped by the expansion of the bubble. Similar dust clumps have been observed at the borders of several IR bubbles (Zavagno et al. 2010; Ji et al. 2012; Liu et al. 2016). 
In this paper, we study these two regions in detail in radio and IR. Section 2 outlines the observation and data reduction of the radio continuum observations. Apart from this, the section also describes the various archival databases used for this study. In Section 4 we discuss the results obtained and Section 5 summarizes the conclusions.

\section{Observation and data reduction}

\subsection{Radio continuum observations}

In order to study the ionized gas component associated with our regions of interest, we carried out radio continuum mapping with the Giant Metrewave Radio Telescope (GMRT), Pune India on 17 and 20 July 2011. GMRT has a hybrid configuration of 30 antennae in a ' $\mathrm{Y}$ ' shaped layout. Each antenna is a parabolic reflecting dish of $45 \mathrm{~m}$ diameter. The central square has 12 randomly placed antennae within a compact area of $1 \times 1 \mathrm{~km}^{2}$ with shortest baselines of $\sim 100 \mathrm{~m}$. This is sensitive to large scale diffuse emission. The remaining 18 antennae are placed six each in the three arms. The largest baseline possible with GMRT is $\sim 25 \mathrm{~km}$ which accounts for the high angular resolution. Details regarding the GMRT configuration can be found in Swarup et al. (1991).

The radio continuum observations were carried out at 1280 and $610 \mathrm{MHz}$ with a bandwidth of $32 \mathrm{MHz}$ in the spectral line mode to minimize the effects of bandwidth smearing and narrowband RFI. Radio sources 3C48 and 3C286 were used as primary flux calibrators and 1626-298 was used as phase calibrator for estimating the amplitude and phase gains for flux and phase calibration of the measured visibilities. Data reduction is performed using the Astronomical Image Processing System (AIPS) using standard procedures. The task TVFLG is used to identify bad data and also channels affected by RFI. The calibrated data was averaged in frequency to the extent to keep the bandwidth smearing effects negligible. The wide-field imaging technique is employed to account for $w$-term effects (non-coplanarity). Several iterations of 'phase-only' self calibration are performed in order to minimize amplitude and phase errors and obtain better rms noise in the maps. The primary beam correction is applied using the task PBCOR.

While observing close to the Galactic plane, the Galactic diffuse emission becomes significant and contributes toward increasing the system temperature which becomes relevant at low frequencies. At the frequencies of our radio observations (especially at $610 \mathrm{MHz}$ ), a rescaling of the final image is essential. To determine the scaling factor at $1280 \mathrm{MHz}$, we follow the general method of estimating the sky temperature, $T_{\text {sky }}$, using the measurements obtained from the the all-sky $408 \mathrm{MHz}$ survey of Haslam et al. (1982). This method assumes the Galactic diffuse emission to

follow a power-law spectrum and $T_{s k y}$ at frequency $\nu$ for the target position is determined using the following equation

$$
T_{s k y}=T_{s k y}^{408}\left(\frac{\nu}{408 \mathrm{MHz}}\right)^{\gamma}
$$

where, $\gamma$ is the spectral index of the Galactic diffuse emission and is taken as -2.55 (Roger et al. 
1999). Using this, we obtain a scaling factor of 1.2 at $1280 \mathrm{MHz}$. For $610 \mathrm{MHz}$, we obtain the scaling factor from the observed self-power of the antennas following the procedure outlined in Marcote et al. (2015). Self-power of each antenna is measured at the position of the flux calibrator and the target at similar elevations. After retaining only the antennas with stable self-power, the ratio of individual data points of S10 and 3C286 is calculated for each antenna and polarization. Median of the ratios removes the outliers and gives a scaling factor of $1.7 \pm 0.02$.

\section{Available data from archives}

\subsection{Mid-infrared data from Spitzer}

Mid-infrared (MIR) data have been obtained from the archives of Spitzer Space Telescope. Photometric data in the four IRAC bands $(3.6,4.5,5.8,8.0 \mu \mathrm{m})$ have been retrieved from the 'highly reliable' catalog of the Galactic Legacy Infrared Midplane Survey Extraordinaire (GLIMPSE) survey (Benjamin et al. 2003). $24 \mu \mathrm{m}$ images have been obtained from the MIPSGAL survey (Rieke et al. 2004). The angular resolution of the images in the IRAC bands are $<2^{\prime \prime}$ whereas it is $\sim 6^{\prime \prime}$ at $24 \mu \mathrm{m}$. These data are used to study the population of young stellar objects (YSOs) and warm dust associated with the regions.

\section{2. $\quad$ Far-infrared data from Herschel}

Far-infrared (FIR) data used in this paper have been obtained from the Herschel Space Observatory archives. Level 2.5 processed $70-500 \mu \mathrm{m}$ images from the Photodetector Array Camera and Spectrometer (PACS; Poglitsch et al. 2010) and Spectral and Photometric Imaging Receiver (SPIRE; Griffin et al. 2010) observed as part of the Herschel infrared Galactic plane Survey (HIGAL; Molinari et al. 2010) in parallel mode are retrieved. Resolutions of the images are 5, 11.4, 17.9, 25 and $35.7^{\prime \prime}$ for $70,160,250,350$ and $500 \mu \mathrm{m}$ respectively. We use the FIR data to study the physical properties of cold dust emission associated with the regions.

\section{3. $\quad 843 \mathrm{MHz}$ data from SUMSS}

The radio map at $843 \mathrm{MHz}$ used in this study is obtained from the Sydney University Molonglo Sky Survey (SUMSS) archives. Details regarding this survey can be found in Mauch et al. (2003). The map has a resolution of $45^{\prime \prime}$ and a pixel size of $11^{\prime \prime}$. SUMSS is similar in sensitivity and resolution to the northern NRAO VLA Sky Survey (NVSS). 


\section{Results and Discussion}

\subsection{Ionized Emission}

For understanding the distribution of ionized gas associated with S10 and EGO345, we generate continuum maps at 610 and $1280 \mathrm{MHz}$ by setting the 'robustness' parameter to +1 (on a scale where +4 represents nearly natural weighting and -4 is close to uniform weighting of the baselines) while running IMAGR. We further use the task UVTAPER to weigh down the long baselines. The above procedures enable us to probe larger spatial scales of the extended diffuse emission in the regions. Figure 3 shows the radio continuum maps overlaid on the $8 \mu \mathrm{m}$ IRAC image. Table 1 gives the details of the observation and the maps.

The region associated with S10 shows the presence of faint diffuse emission mostly distributed in the second quadrant in the interior of the bubble. The $610 \mathrm{MHz}$ emission displays a relatively steep density gradient with enhanced emission towards the likely centre of the bubble and a more extended emission towards the north-east. However, the higher frequency map at $1280 \mathrm{MHz}$ is seen to be less extended in the south-east and north-west direction but follows the general morphology seen at $610 \mathrm{MHz}$. The radio contours near the centre are enveloped in the south-west direction by an arc-type $8 \mu \mathrm{m}$ structure. Apart from this, in the $1280 \mathrm{MHz}$ map we see ionized emission beyond the west periphery of the bubble. This emission is not detected in the $610 \mathrm{MHz}$ map down to the $3 \sigma$ level. This could be due to a combination of the nature of the ISM there as well as the lower sensitivity achieved at $610 \mathrm{MHz}$. It is difficult to comment on the association of this detached emission with that of the bubble. For the region associated with EGO345, the $610 \mathrm{MHz}$

Table 1: Details of the radio interferometric continuum observations.

\begin{tabular}{lll}
\hline \hline Details & $610 \mathrm{MHz}$ & $1280 \mathrm{MHz}$ \\
\hline Date of Obs. & $17 \mathrm{July} 2011$ & 20 July 2011 \\
Flux Calibrators & 3C286,3C48 & 3C286,3C48 \\
Phase Calibrators & $1626-298$ & $1626-298$ \\
Synth. beam & $14.4^{\prime \prime} \times 8.5^{\prime \prime}$ & $8.8^{\prime \prime} \times 4.4^{\prime \prime}$ \\
Position angle. (deg) & 10.61 & 15.02 \\
rms noise (mJy/beam) & 0.7 & 0.2 \\
Int. Flux (mJy) & $203(\mathrm{~S} 10)$ & $44(\mathrm{~S} 10)$ \\
(integrated upto $3 \sigma$ level) & $43($ EGO345) & $132($ EGO345) \\
\hline
\end{tabular}

map shows a smooth and nearly spherical morphology with the $8 \mu \mathrm{m}$ triangular shaped emission located towards its lower half. The $1280 \mathrm{MHz}$ map shows a relatively clumpier morphology which is more extended in the north-east and south-west direction as compared to the $610 \mathrm{MHz}$ emission. The position of peak flux density lies $\sim 24^{\prime \prime}$ north-east of the position of the EGO.

For optically thin and free-free emission, the excitation parameter, $u$, and the total flux of 

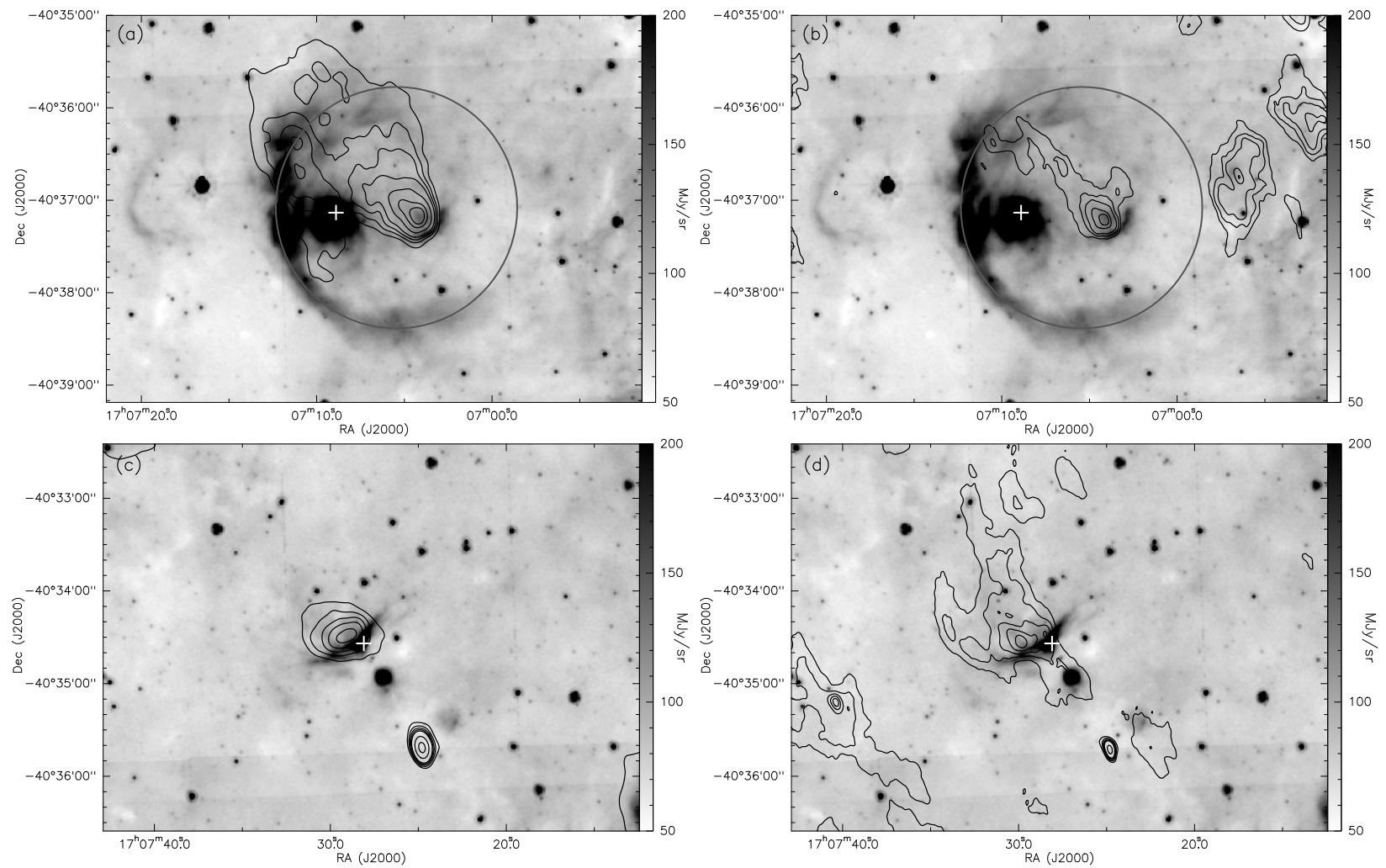

Fig. 3.- Radio continuum emission probed in both the regions over plotted on the IRAC $8.0 \mu \mathrm{m}$ images. (a) $610 \mathrm{MHz}$ map of the region associated with S10. The contour levels are 3, 3.5, 4.0, 4.5, 5.5, 6.5, 7 times $\sigma(0.7 \mathrm{mJy} /$ beam $)$. (b) $1280 \mathrm{MHz}$ of the region associated with $\mathrm{S} 10$. The contour levels are 3, 4, 5, 7, 8 times $\sigma(0.2 \mathrm{mJy} / \mathrm{beam})$. (c) Same as (a) but for the region associated with EGO345. The contour levels are 3, 4, 5, 6, 7 times $\sigma$. (d) Same as (b) but for the region associated with EGO345. The contour levels are 3, 4, 5, 7, 9, 13 times $\sigma$. The circles in (a) and (b) shows the extent of the bubble S10. The '+' marks indicate the position of the IRAS point sources associated with both the regions. 
ionizing Lyman continuum photons, $N_{\text {luc }}$, at a given frequency, $\nu$, can be estimated using the following formulation from Schraml \& Mezger (1969) and Panagia (1973),

$$
\begin{aligned}
{\left[\frac{u}{\mathrm{pc} \mathrm{cm}^{2}}\right]=4.5526 } & {\left[a\left(\nu, T_{e}\right)^{-1}\left[\frac{\nu}{\mathrm{GHz}}\right]^{0.1}\left[\frac{T_{e}}{\mathrm{~K}}\right]^{0.35}\left[\frac{S}{\mathrm{Jy}}\right]\left[\frac{D}{\mathrm{kpc}}\right]^{2}\right]^{\frac{1}{3}} } \\
u & =2.01 \times 10^{-19}\left[\frac{N_{l y c}}{\beta_{\mathrm{RR}}}\right]^{\frac{1}{3}} \mathrm{pc} \mathrm{cm}^{-2}
\end{aligned}
$$

where, $a\left(\nu, T_{e}\right)$ is the correction factor taken as 0.99 (Mezger \& Henderson 1967), $T_{e}$ is the electron temperature, $S$ the integrated flux density and $D$ the distance to the source. $\beta_{\mathrm{RR}}$ is the recombination rate to the excited levels of hydrogen which is assumed to be $3.43 \times 10^{-13}$ for an electron temperature of $7000 \mathrm{~K}$ (Panagia 1973). We determine $T_{e}$ using the Galactic temperature gradient relation given in Deharveng et al. (2000). The Galactocentric distance to our regions is determined to be $2.8 \mathrm{kpc}$ using the expression given in Xue et al. (2008). This Galactocentric distance corresponds to $T_{e}$ of $5300 \mathrm{~K}$. To account for the corresponding value of $\beta_{\mathrm{RR}}$ for this temperature, we have applied a scaling factor of 1.0976 to Equation 3 as discussed in Panagia (1973).

As discussed in Churchwell et al. (2006), the probability of chance alignments of bubbles with HII regions is very small $(<1 \%)$, hence the detected ionized emission can be assumed to be due to the massive star(s) driving the bubble S10. To determine the excitation parameter, total flux of ionizing Lyman continuum photons and the spectral type of the ionizing source responsible for the bubble S10, we assume the emission to be free-free and optically thin at $1280 \mathrm{MHz}$. We integrate the flux densities upto $3 \sigma$ level and plug in the values in Equations 2 and 3. For an integrated flux density of $44 \mathrm{mJy}$ and an electron temperature, $T_{e}$, of $5300 \mathrm{~K}$, we derive values of $13.3 \mathrm{pc} \mathrm{cm}^{-2}$ for the excitation parameter $(u)$ and 47.0 for the logarithm of ionizing Lyman continuum photon $\left(\log N_{l y c}\right)$. Assuming a single exciting source responsible for the ionized emission, we estimate the ZAMS spectral type to lie between B0.5 - B0 (see Table II of Panagia (1973)). This estimate is with the assumption of optically thin emission and hence serves as a lower limit as the emission could be optically thick at $1280 \mathrm{MHz}$. Various studies in the literature have shown that dust absorption of Lyman continuum photons can be very high (Inoue et al. 2001; Arthur et al. 2004; Paron et al. 2011). With limited knowledge of the dust properties, we have not accounted for the dust absorption here. We determine the spectral index, $\alpha$ defined by $S \propto \nu^{\alpha}$ using the peak flux densities from the two maps after convolving the $1280 \mathrm{MHz}$ map to the resolution of the $610 \mathrm{MHz}$ map $\left(14.4^{\prime \prime} \times 8.5^{\prime \prime}\right)$. The estimated spectral index of -0.1 is consistent with what is expected from optically thin freefree emission. Close to the radio peak $\left(\alpha_{2000}=17: 07: 04.20, \delta_{2000}=-40: 37: 11.00\right)$, there is a red NIR source (hereafter IRS1) $\left(\alpha_{2000}=17: 07: 03.60, \delta_{2000}=-40: 37: 10.70\right)$ with colours J $\mathrm{H}=2.44$ and $\mathrm{H}-\mathrm{K}=1.67$. The nature of this source will be discussed later to ascertain whether it is the NIR counterpart of the ionizing star.

Using the integrated flux density of $132 \mathrm{mJy}$ at $1280 \mathrm{MHz}$ and following the above formulation, we also estimate the physical parameters for the region associated with EGO345. The excitation 
parameter, total flux of ionizing Lyman continuum photon and spectral type range is determined to be $18.9 \mathrm{pc} \mathrm{cm}^{-2}, 47.45$ and $\mathrm{B} 0$ - O9.5, respectively. It should be noted here that the peaks at 610 and $1280 \mathrm{MHz}$ are offset from each other by $\sim 10^{\prime \prime}$. A possible reason for this offset could be the nature of the ISM in this region. If there is an inhomogeneous density distribution then it could lead to varying optical thickness. EGOs are known to harbour outflows and jets, hence one would also expect thermal emission from jets giving rise to positive spectral indices. Shock-induced non-thermal emission could also co-exist in such environments.

Apart from S10 and EGO345, the radio maps (Figure 3) show the presence of a relatively strong radio emitting region $\sim 1^{\prime}$ to the south-west of the position of EGO345 with integrated flux densities of 21 and $7.5 \mathrm{mJy}$ and peak flux densities of 16.9 and $6.5 \mathrm{mJy} /$ beam at 610 and $1280 \mathrm{MHz}$, respectively. From the peak flux density values we infer the associated emission to be non-thermal with a steep negative spectral index of -1.3. It is unclear whether this emission is associated with EGO345. No counterpart is reported in NED or Simbad. The SUMSS map shows a faint blob coincident with the location of this source.

\subsection{Population of Young Stellar Objects}

In order understand the stellar population and probe the star forming activity in the two regions, we identify and classify the associated young stellar objects (YSOs). Infrared colors have been proven to be a powerful tool for the identification of YSOs (Allen et al. 2004; Simon et al. 2007; Gutermuth et al. 2008). We have used the GLIMPSE 'highly reliable' catalog to retrieve the IRAC band magnitudes within $120^{\prime \prime}$ of the expected centre of the bubble $\left(\alpha_{2000}=17: 07: 05.45\right.$, $\left.\delta_{2000}=-40: 37: 04.80\right)$ and within $60^{\prime \prime}$ centered on the position of EGO345 $\left(\alpha_{2000}=17: 07: 27.60\right.$, $\left.\delta_{2000}=-40: 34: 45.00\right)$. We retrieved 65 and 23 sources with good quality data in all IRAC bands for the regions associated with S10 and EGO345, respectively. The red source IRS1 has photometric magnitudes available in the first three IRAC bands only. Using IRAF task qphot, we estimate its magnitude at $8 \mu \mathrm{m}$. Using the IRAC colours we have identified YSOs in our field adopting the procedures followed by these authors, the details of which are outlined are below:

1. Based on the IRAC colours of the models of protostellar envelopes (Class I) and protoplanetary disks (Class II) described in Allen et al. (2004), we identified regions on the [3.6] - [4.5] vs [5.8] - [8.0] colour-colour plot (CCP) to isolate the Class I and II YSOs. Figure 4 shows the CCP where the boxes drawn to demarcate the regions occupied by Class I and Class II models are adopted from Vig et al. (2007). Using this method we have identified 10 candidate YSOs out of which 6 are Class I, one is Class II and 3 are either Class I/II type of sources in the S10 region. IRS1 falls in the region for Class I YSOs. One candidate YSO of either Class I/II type is identified in the region associated with EGO345.

2. Simon et al. (2007) have proposed a set of criteria based on the IRAC colours for the identification of YSOs which includes removal of contaminants like galaxies, PAH sources. These 
criteria does not differentiate between Class I and II YSOs. The colour cuts adopted are

$$
\begin{aligned}
& {[3.6]-[4.5]>0.6 \times([4.5]-[8.0])-1.0} \\
& {[4.5]-[8.0]<2.8} \\
& {[3.6]-[4.5]<0.6 \times([4.5]-[8.0])+0.3} \\
& {[3.6]-[4.5]>-([4.5]-[8.0])+0.85}
\end{aligned}
$$

In Figure 4, we show the location of YSOs in the CCP based on the above equations. Using this method we have identified 11 candidate YSOs including IRS1 in the region S10 and 3 candidate YSOs in the region EGO345.

3. Gutermuth et al. (2008) have used the [4.5] - [5.8] colour for identifying YSOs. They use various criteria based on the IRAC colours to remove contaminants such as PAH dominated galaxies, AGNs and sources dominated by shock emission. This ensures a confident YSO sample.

(i) Sources are likely protostars (Class I) if they have an extremely red discriminant colour $([4.5]-[5.8]>1)$. Sources having moderate red discriminant colour $(0.7<[4.5]-[5.8]$ $\leqslant 1)$ and $[3.6]-[4.5]>0.7$ are also considered as likely protostars.

(ii) Class II sources satisfy

$$
\begin{aligned}
& {[4.5]-[8.0]>0.5} \\
& {[3.6]-[5.8]>0.35} \\
& {[3.6]-[5.8] \leqslant \frac{0.14}{0.04} \times([[4.5]-[8.0]]-0.5)+0.5}
\end{aligned}
$$

Location of protostars (Class I) and Class II sources following the criteria by Gutermuth et al. (2008) is shown in Figure 4. Using this we have detected 11 candidate YSOs out of which 4 are likely protostars (Class I) and the rest including IRS1 are Class II type sources in the region S10 and 4 Class II type YSOs in the region EGO345.

Adopting the various criteria described above, we have identified 14 YSOs including IRS1 in the region associated with S10 and 5 YSOs in the region associated with EGO345. Table 2 lists the identified YSOs in S10 and EGO345. In Figure 5, we show the spatial distribution of the identified YSOs overplotted on the $8 \mu \mathrm{m}$ image. In the figure, we mark the location of two additional sources which are listed as extreme red sources in Robitaille et al. (2008). The distribution of the identified YSOs are mostly towards the western part of the bubble and the north-eastern part of EGO345. It should be kept in mind that the identified YSOs are a sub-sample given the fact that we are concentrating only on those detected in all four IRAC bands.

\subsection{Nature of IRS1}

As discussed in the previous section, IRS1 is a likely Class I (Allen et al. 2004) or Class II (Gutermuth et al. 2008) YSO. It is located $\sim 7^{\prime \prime}$ westward from the peak position of the ionized 
Table 2: List of YSOs detected in S10 and EGO345 based on the three classification schemes.

\begin{tabular}{cccccc}
\hline Source & $\begin{array}{c}\text { RA (J2000) } \\
\text { (hh:mm:ss.ss) }\end{array}$ & $\begin{array}{c}\text { DEC (J2000) } \\
\text { (dd:mm:ss.ss) }\end{array}$ & Allen et al. (2004) & Gutermuth et al. (2008) & Simon et al. (2007) \\
\hline & & & YSOs in S10 & & \\
\hline 1 & $17: 06: 55.51$ & $-40: 36: 46.33$ & Class I/II & Class II & YSO \\
$2^{\dagger}$ & $17: 06: 56.03$ & $-40: 37: 39.68$ & Class I/II & Class I & YSO \\
3 & $17: 06: 57.34$ & $-40: 37: 28.67$ & Class I & Class I & - \\
4 & $17: 06: 58.25$ & $-40: 36: 44.42$ & Class II & Class I & - \\
5 & $17: 06: 58.26$ & $-40: 36: 15.30$ & - & Class I & YSO \\
6 & $17: 06: 58.57$ & $-40: 37: 58.73$ & Class I & Class II & YSO \\
7 & $17: 07: 03.43$ & $-40: 36: 32.22$ & - & Class II & YSO \\
$8^{*}$ & $17: 07: 03.60$ & $-40: 37: 10.70$ & Class I & Class II & YSO \\
$9^{\dagger}$ & $17: 07: 03.84$ & $-40: 37: 48.76$ & Class I & YSO \\
10 & $17: 07: 05.56$ & $-40: 36: 37.40$ & - & Class II & YSO \\
11 & $17: 07: 06.63$ & $-40: 36: 26.24$ & Class I/II & - & YSO \\
12 & $17: 07: 10.66$ & $-40: 38: 44.02$ & - & Class II & YSO \\
13 & $17: 07: 11.98$ & $-40: 37: 06.42$ & Class I & Class II & YSO \\
14 & $17: 07: 14.77$ & $-40: 36: 15.84$ & Class I & & YSO \\
\hline & & & YSOs in EGO345 & YSO \\
\hline 1 & $17: 07: 25.85$ & $-40: 34: 03.97$ & - & Class II & YSO \\
2 & $17: 07: 29.23$ & $-40: 33: 54.29$ & - & Class II & - \\
3 & $17: 07: 31.10$ & $-40: 34: 47.86$ & Class I/II & Class II & Class II \\
4 & $17: 07: 31.66$ & $-40: 35: 12.88$ & - & & -
\end{tabular}

${ }^{*}$ The NIR source IRS1; ${ }^{\dagger}$ extreme red sources from Robitaille et al. (2008) 

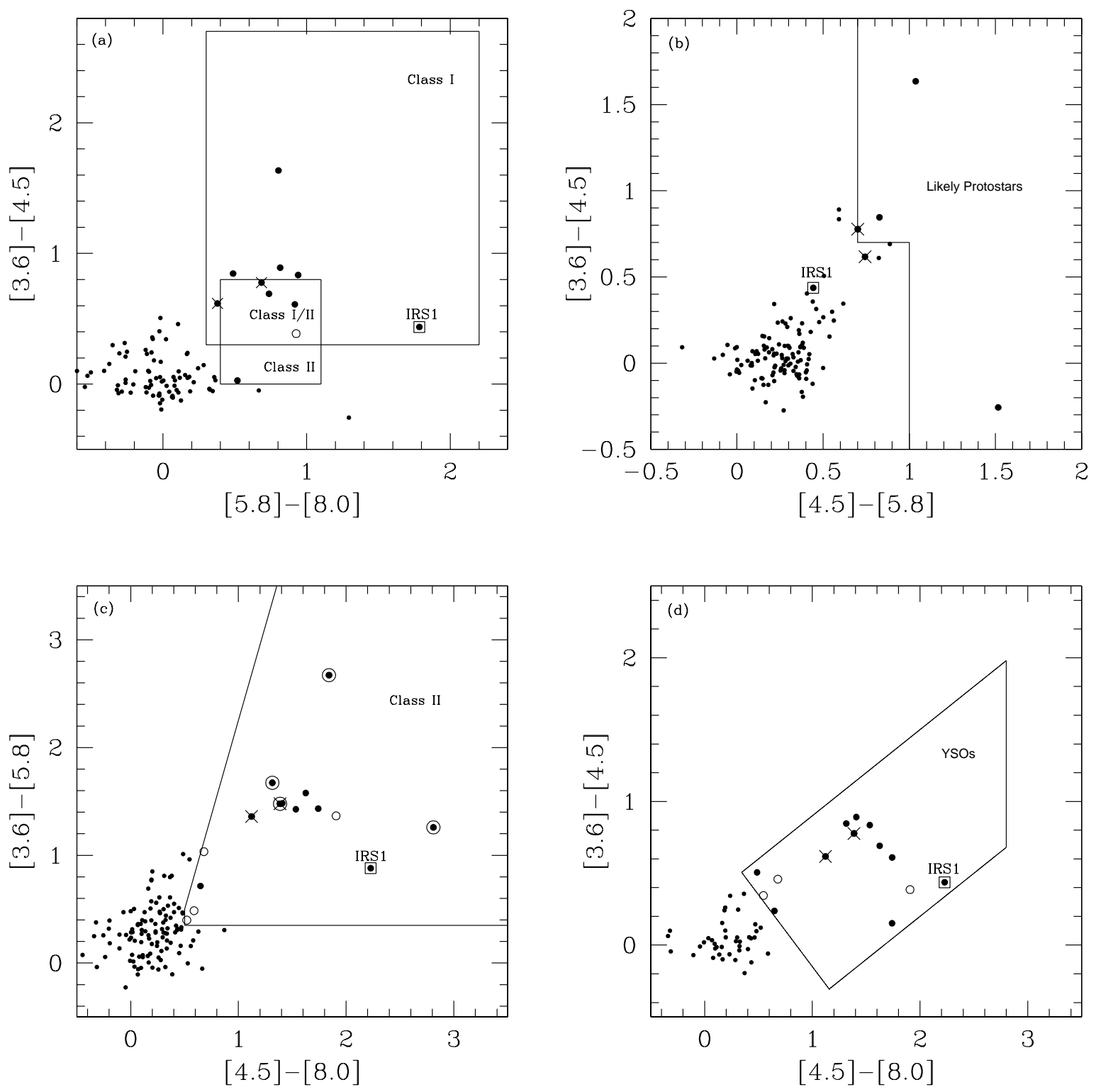

Fig. 4.- IRAC CCPs describing the various criteria discussed in the text. YSOs identified in the regions associated with S10 and EGO345 are shown as filled and open circles, respectively. Crosses denote the two extreme red sources identified in Robitaille et al. (2008). Location of IRS1 is also highlighted with an overplotted open square. (a) YSO identification as per criteria discussed in Allen et al. (2004). The boxes to demarcate the location of Class I (larger box) and Class II (smaller box) are adopted from Vig et al. (2007). Sources falling in the overlapping area are designated as Class I/II. (b) Criteria following Gutermuth et al. (2008). The region occupied by likely protostars (Class I) is shown. (c) Criteria for Class II sources following the method of Gutermuth et al. (2008). The four protostars (Class I) sources identified in (b) are also marked with overplotted open circles. (d) Criteria adopted from Simon et al. (2007). 


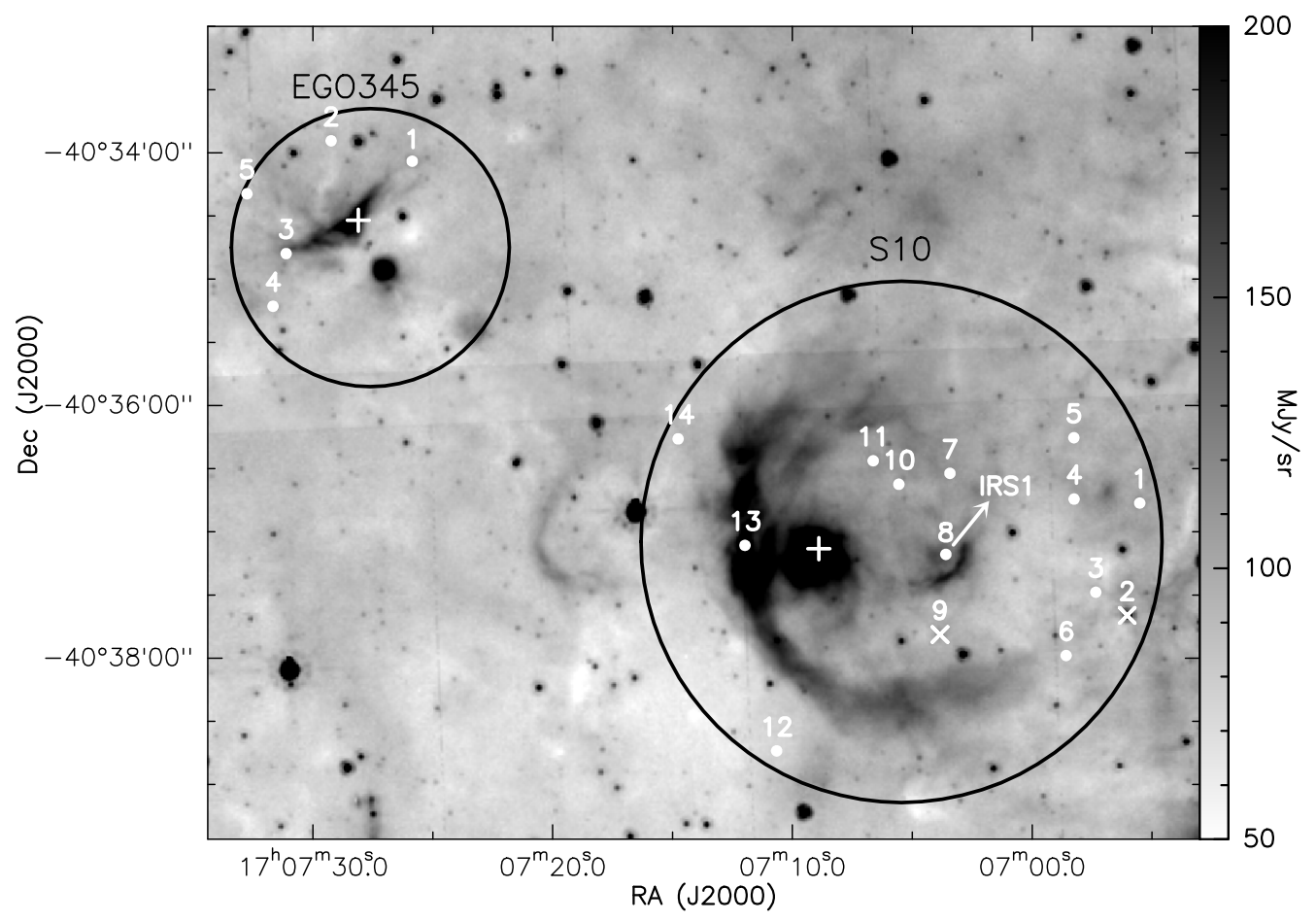

Fig. 5.- YSOs (white filled circles) identified by the various methods discussed in the text are marked over the $8.0 \mu \mathrm{m}$ image. The '+' marks show position of IRAS 17036-4033 and IRAS 17039-4030 in the regions associated with S10 and EGO 345, respectively. The cross marks are the extreme red sources identified by Robitaille et al. (2008). The position of IRS1 is highlighted. 
emission probed in the radio frequencies. To derive the physical parameters of IRS1 we have carried out Spectral Energy Distribution (SED) modelling using the online SED fitting tool of Robitaille et al. (2007). The basic models are computed using Monte Carlo based radiative transfer algorithm which uses various combinations of central star, disk, infalling envelope, and cavities carved out by bipolar outflows. A reasonably large parameter space is explored in these models. Assuming that IRS1 is associated with the bubble S10, we have used a distance range of 5.5 to 5.9 $\mathrm{kpc}$ in the model fitting tool. As discussed in Section 4.1, IRS1 is a reddened source and its location in the JHK CCP (not presented in the paper) gives an estimate of $A_{v} \sim 15$ mags. Hence, for the model fitting we use a range of $A_{v}=1$ - 20 mags. Apart from the MIR fluxes, we use the NIR JHK fluxes from 2MASS1 1 . IRS1 is enclosed within a FIR clump (discussed later). Taking the retrieved clump aperture with an effective diameter of $\sim 12^{\prime \prime}$, we extract the flux densities at 24, 70, 160, $250,350,500,870$, and $1200 \mu \mathrm{m}$ and use them as upper limits for the SED fits. The $870 \mu \mathrm{m}$ and $1200 \mu \mathrm{m}$ data are from ATLASGAL 2 survey and Beltrán et al. (2006), respectively. The model SED is generated using 7 data points and 8 upper limits. We have assumed a conservative $10 \%$ error on the used flux densities. In Figure 6, we show the model fits for IRS1 satisfying the criteria $\chi^{2}-\chi_{\text {best }}^{2}$ (per data point) $<3$. The weighted average (weight is taken as $1 / \chi^{2}$ ) of the physical parameters retrieved from the above best fitting models are listed in Table 3 with the values obtained for the best fit given in parenthesis. The best fit model gives the mass estimate of the source as $6.2 \mathrm{M}_{\odot}$ which suggests IRS1 to be an intermediate-mass star. IRS1 is therefore unlikely to be the NIR counterpart of the exciting B0.5 - B0 star responsible for the ionized emission. It is possible that the massive ionizing star is deeply embedded and does not reveal itself in the NIR. It should however be noted that these values of the parameters are to be taken with caution since we are dealing with a large parameter space with very few data points to constrain the models.

\subsection{Emission from dust component}

\subsubsection{Temperature and column density maps}

Emission from dust continuum in the regions associated with S10 and EGO345 is shown in Figure 7. Warm dust is seen in localized areas near the bubble and the EGO whereas, the cold dust emission is seen to be distributed in a diagonal stretch along the north-east and south-west direction.

The Raleigh-Jeans part of the thermal emission from cold dust is covered by the Herschel FIR

\footnotetext{
${ }^{1}$ This publication makes use of data products from the Two Micron All Sky Survey, which is a joint project of the University of Massachusetts and the Infrared Processing and Analysis Center/California Institute of Technology, funded by the NASA and the NSF.

${ }^{2}$ This project is a collaboration between the Max Planck Gesellschaft (MPG: Max Planck Institute fr Radioastronomie, MPIfR Bonn, and Max Planck Institute for Astronomie, MPIA Heidelberg), the European Southern Observatory (ESO) and the Universidad de Chile
} 


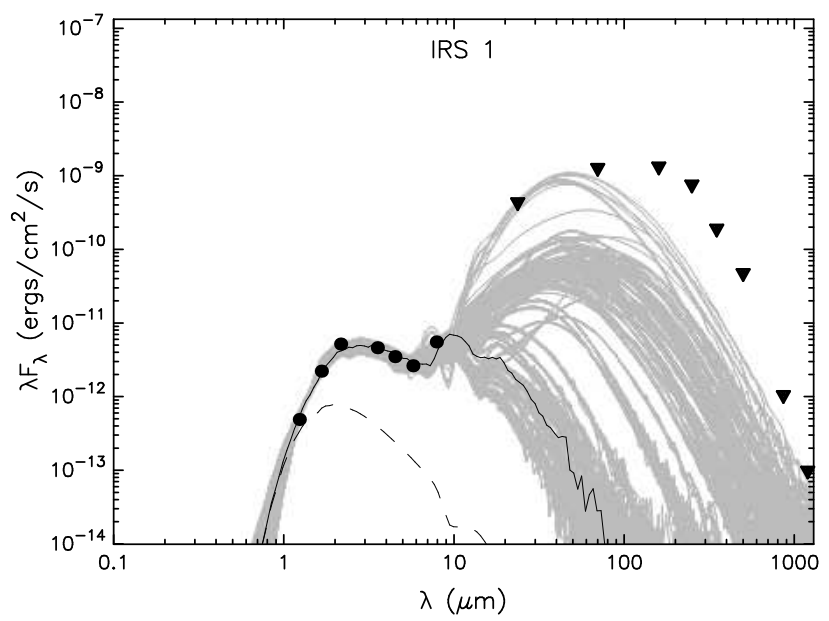

Fig. 6. - Best fit SED models of IRS1 using the online tool of Robitaille et al. (2007). NIR and MIR fluxes are shown as solid circles. Flux densities for MIPSGAL $24 \mu \mathrm{m}$, PACS 70, $160 \mu \mathrm{m}$, SPIRE 250, 350, $500 \mu \mathrm{m}$, ATLASGAL $870 \mu \mathrm{m}$ and $1200 \mu \mathrm{m}$ are given as upper limits (filled triangles). The best fit model is shown as solid black line. The plots shown in grey are the models satisfying the criteria $\chi^{2}-\chi_{\text {best }}^{2}$ (per data point) $<3$. The photosphere of central source is shown as the dashed curve (with interstellar extinction but with absence of circumstellar dust).

bands $(160-500 \mu \mathrm{m})$. Hence, we use the Herschel data to study the physical properties of the cold dust emission associated with both the regions. We generate the temperature and the column density maps using a pixel-by-pixel SED modelling of the dust emission to a gray / modified black body. In generating the maps, we have excluded $70 \mu \mathrm{m}$ data since this band has contribution from both warm and cold dust. Hence, a single modified blackbody model would possibly overestimate the cold dust temperatures and a two-temperature gray body is therefore essential to represent the emission from $70 \mu \mathrm{m}$ (Galametz et al. 2012). Prior to the SED modelling, the following preliminary steps are carried out using the the Herschel data compatible software HIPE 3

1. Using the task 'Convert Image Unit', the image units of the SPIRE images $\left(\mathrm{MJy} \mathrm{Sr}^{-1}\right)$ are converted to a common surface brightness unit of $\mathrm{Jy}_{\mathrm{pixe}}{ }^{-1}$ of the PACS images.

2. The plug-in 'Photometric Convolution' is then used to project all the images onto a common grid with the same pixel size and resolution of $14^{\prime \prime}$ and $35.7^{\prime \prime}$, respectively, which are the parameters of the $500 \mu \mathrm{m}$ image (lowest among the four bands).

Subsequent to this, we model the dust emission in each pixel to a modified blackbody using

\footnotetext{
${ }^{3}$ The software package for Herschel Interactive Processing Environment (HIPE) is the application that allows users to work with the Herschel data, including finding the data products, interactive analysis, plotting of data, and data manipulation.
} 


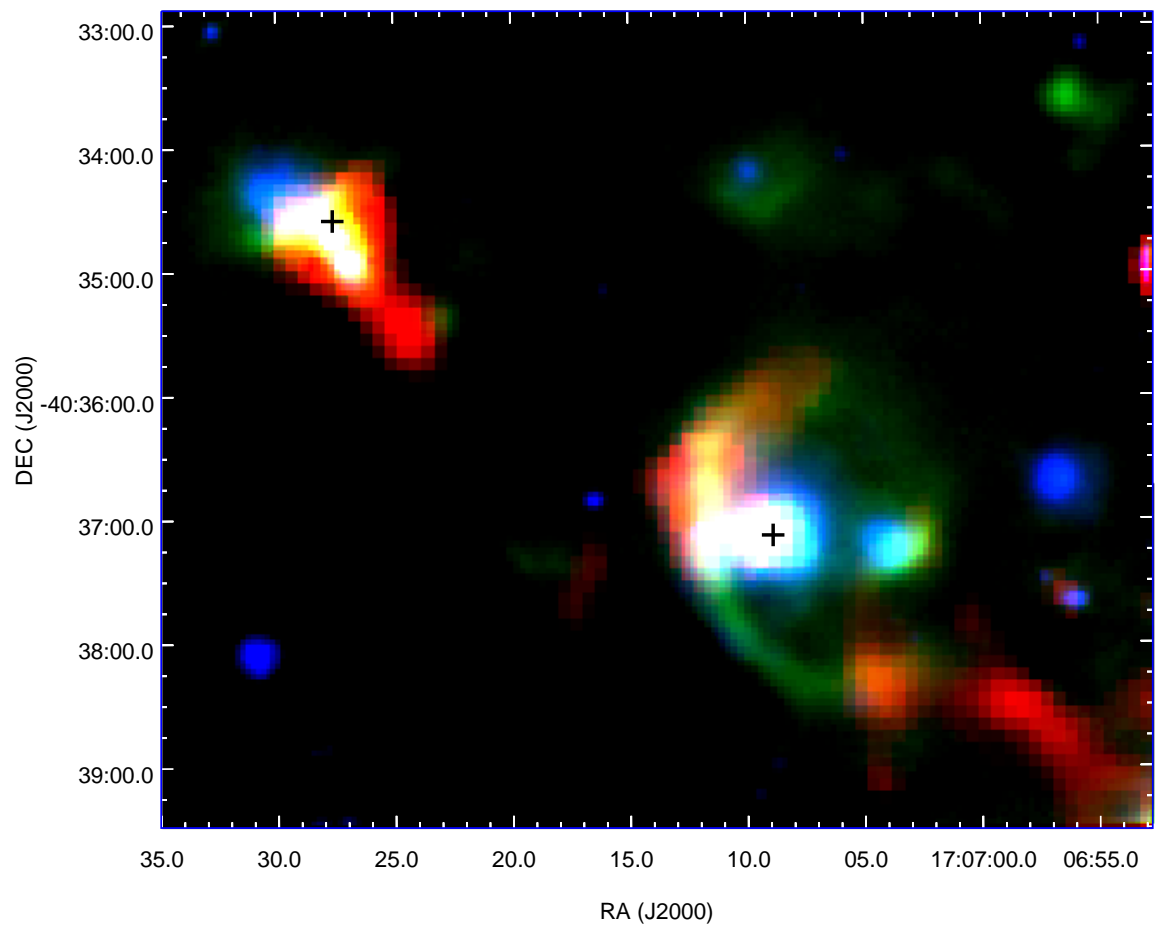

Fig. 7.- Three-color composite image of the regions associated with S10 and EGO345 with $24 \mu \mathrm{m}$ Spitzer- MIPSGAL (blue), $70 \mu \mathrm{m}$ Herschel-PACS (green), $250 \mu \mathrm{m}$ Herschel-SPIRE (red). 
Table 3: Weighted mean of the physical parameters for IRS1 retrieved from the SED modelling. Values in the parenthesis are from the best fit model. Second row lists the range for each parameter fitted by all the models satisfying $\chi^{2}-\chi_{\text {best }}^{2}$ (per data point) $<3$.

\begin{tabular}{|c|c|c|c|c|c|c|c|}
\hline $\begin{array}{c}\log t_{*} \\
(y r)\end{array}$ & $\begin{array}{l}\text { Mass } \\
\left(\mathrm{M}_{\odot}\right)\end{array}$ & $\begin{array}{c}\log \mathrm{M}_{\text {disk }} \\
\left(\mathrm{M}_{\odot}\right)\end{array}$ & $\begin{array}{c}\log \dot{\mathrm{M}}_{\text {disk }} \\
\left(\mathrm{M}_{\odot} y r^{-1}\right)\end{array}$ & $\begin{array}{c}\log \mathrm{M}_{e n v} \\
\left(\mathrm{M}_{\odot}\right)\end{array}$ & $\begin{array}{c}\log \mathrm{T}_{*} \\
(\mathrm{~K})\end{array}$ & $\begin{array}{c}\log \mathrm{L}_{\text {total }} \\
\quad\left(\mathrm{L}_{\odot}\right)\end{array}$ & $\begin{array}{c}\mathrm{A}_{V} \\
(\mathrm{mag})\end{array}$ \\
\hline $5.70(6.19)$ & $4.75(6.24)$ & $-4.02(-6.70)$ & $-9.50(-12.04)$ & $-2.46(-5.60)$ & $3.96(4.28)$ & $2.42(3.31)$ & $14.29(14.33)$ \\
\hline $3.03-7.00$ & $1.04-10.23$ & $-7.13--0.17$ & $-13.51--4.36$ & $-7.67-2.65$ & $3.60-4.32$ & $1.43-3.37$ & $2.92-20.00$ \\
\hline
\end{tabular}

the following expression (Ward-Thompson \& Robson 1990; Faimali et al. 2012; Pitann et al. 2013; Mallick et al. 2015),

$$
S_{\nu}(\nu)-I_{b k g}(\nu)=B_{\nu}\left(\nu, T_{d}\right) \Omega\left(1-e^{-\tau_{\nu}}\right)
$$

where, $S_{\nu}(\nu)$ is the observed flux density, $I_{b k g}(\nu)$ is the background flux which in our case is obtained from the Gaussian fit explained below, $B_{\nu}\left(\nu, T_{d}\right)$ is the Planck's function, $T_{d}$ is the dust temperature, $\Omega$ is the solid angle (in steradians) from where the flux is obtained (solid angle subtended by a $14^{\prime \prime} \times 14^{\prime \prime}$ pixel) and $\tau_{\nu}$ is the optical depth. The optical depth in turn is given by,

$$
\tau_{\nu}=\mu_{\mathrm{H}_{2}} m_{\mathrm{H}} \kappa_{\nu} N\left(\mathrm{H}_{2}\right)
$$

where, $\mu_{\mathrm{H}_{2}}$ is the mean molecular weight, $m_{\mathrm{H}}$ is the mass of hydrogen atom, $\kappa_{\nu}$ is the dust opacity and $N\left(\mathrm{H}_{2}\right)$ is the column density. We assume a value of 2.8 for $\mu_{\mathrm{H}_{2}}$ (Kauffmann et al. 2008). The dust opacity $\kappa_{\nu}$ is defined to be $\kappa_{\nu}=0.1(\nu / 1000 \mathrm{GHz})^{\beta} \mathrm{cm}^{2} / \mathrm{g}$. $\beta$ is the dust emissivity spectral index which is assumed to be 2 (Hildebrand 1983; Beckwith et al. 1990; André et al. 2010).

The background flux density, $I_{b k g}$ is estimated from a relatively 'smooth' (free of clumpy emission) and 'dark' (free of bright dust emission) region. This is done by visual inspection. We select a region $\sim 1^{\circ}$ away from S10 and EGO345. The background fluxes in the four bands are estimated by fitting a Gaussian to the distribution of individual pixel values in the selected region (Launhardt et al. 2013; Battersby et al. 2011; Mallick et al. 2015). The fitting is done iteratively by rejecting the pixel values outside $\pm 2 \sigma$, until the fit converges to a value. The resultant background flux levels at 160, 250, 350 and $500 \mu \mathrm{m}$ are $-3.22,1.45,0.72,0.26 \mathrm{Jy} \mathrm{pixel}^{-1}$, respectively. The negative flux value at $160 \mu \mathrm{m}$ is due to the arbitrary scaling of the PACS images. The SED modeling is then carried out using non-linear least square Levenberg-Marquardt algorithm pixel wise. We use a conservative $15 \%$ uncertainty on the background subtracted flux densities (Launhardt et al. 2013). Dust temperature and column density are taken as free parameters in the code. From the best fit values, the temperature and column density maps are generated and shown in Figure 8 .

The temperature map shows two peaks $(\sim 23 \mathrm{~K})$ close to the IRAS point sources in the two 


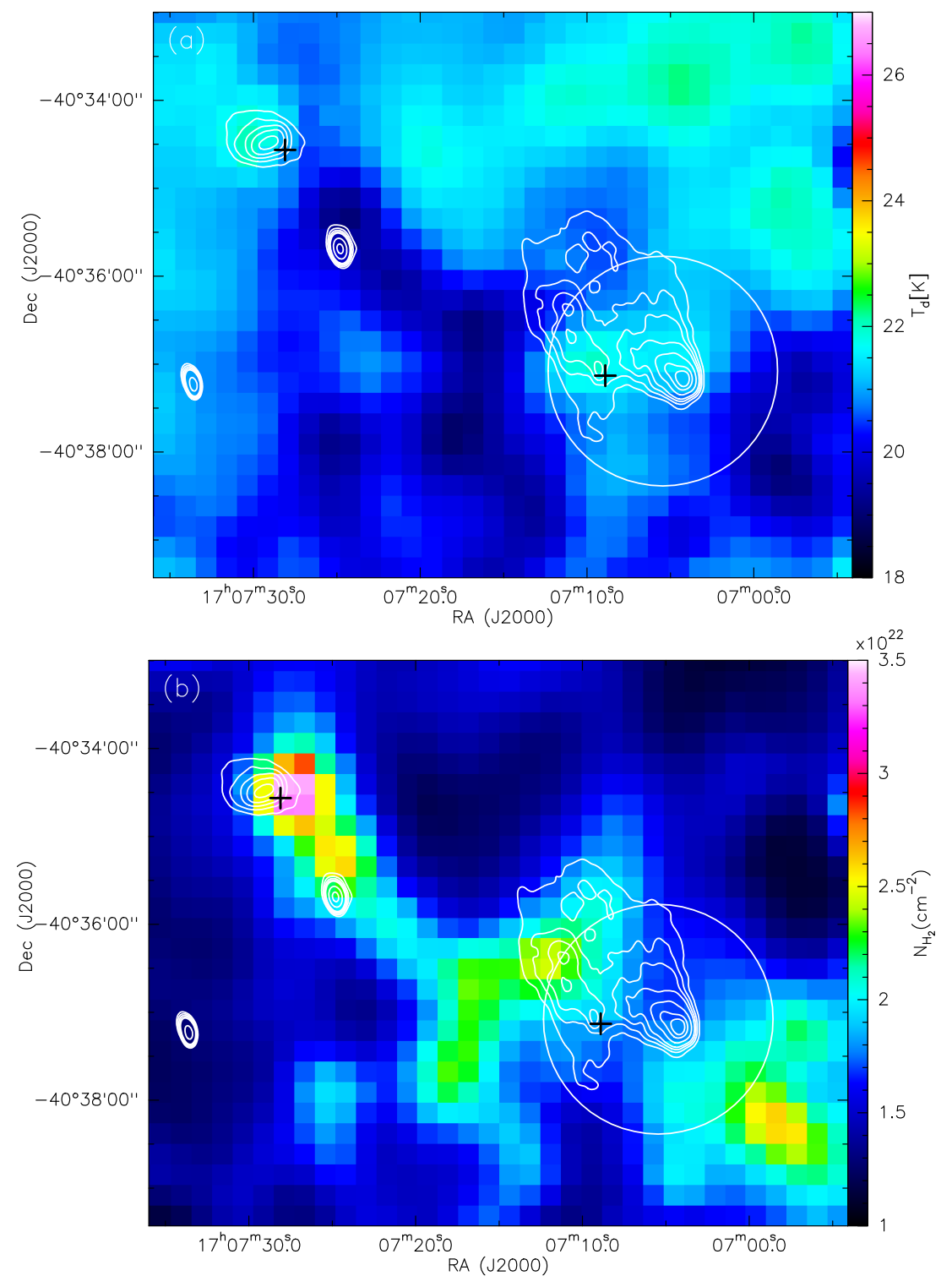

Fig. 8.- (a) Dust temperature map and (b) column density map of regions associated with S10 and EGO345. The radio emission at $610 \mathrm{MHz}$ is also shown as contours with the same levels as in Fig. 3. The white circle shows the extent of the bubble S10. The ' + ' symbols mark the position of IRAS point sources associated with the regions. 
regions. From the overlay of radio contours, it is evident that the ionized regions are traced by warmer dust component compared to the other regions of the map. The peak temperature positions are $\sim 1^{\prime}$ and $24^{\prime \prime}$ towards north-east of the radio peaks in region S10 and EGO345, respectively. The column density map for the region associated with S10 shows a high density elongated clump towards the south-west of the bubble mostly outside the periphery. A high density region is also seen stretching in the south-east and north-west direction on the opposite periphery. The column density map also shows a dense clump associated with the EGO345 region. Another dense clump is seen towards the south-west of EGO345 and north of the position of the bright radio emitting region mentioned in Section 4.1, Apart from this an extended filamentary structure is seen connecting the two regions.

\subsubsection{Properties of dust clumps}

The resolution of the column density map is low $\left(35.7^{\prime \prime}\right)$ and hence does not allow us to detect sub-structures in the map. In order to identify dust clumps or condensations associated with the region around S10 and EGO345, we use the $250 \mu \mathrm{m}$ image which has a optimum resolution of $18^{\prime \prime}$. The threshold for detecting the clump peaks was set to $1.9 \mathrm{Jy}_{\text {pixel }}^{-1}(=20 \sigma)$ to avoid spurious clump detection. The positions of peak intensities in the map are determined by identifying the pixels having the highest value in $3 \times 3$ pixel matrices, with flux values above the estimated threshold. Subsequent to the peak identification, contours are generated to isolate the clumps around these peaks. Using these generated contour levels in the $2 \mathrm{D}$ variation of the clumpfind algorithm (Williams et al. 1994), we detect a total of eight clumps (six in region S10 and 2 in region EGO345). Figure 9 shows the clumps detected using the $250 \mu \mathrm{m}$ image overlaid on $24 \mu \mathrm{m}$ Spitzer-MIPS and the five Herschel bands. In Figure 9(a), we also show the six $1.2 \mathrm{~mm}$ clumps of Beltrán et al. (2006). As seen from the figure, there is an overall overlap of the clumps detected in this work and those from Beltrán et al. (2006). The different numbers, shapes, and sizes of the clumps could be attributed to the different wavelength of the maps and the threshold and contour spacing adopted. The above reason would mostly justify the non-detection of Clumps 4, 5, and 6 by Beltrán et al. (2006).

We determine the masses of the clumps from the column density as well as the $250 \mu \mathrm{m}$ maps. The expressions used are outlined below:

1. From column density map: The masses of the clumps are estimated by determining the mass in each pixel and then summing over all the pixels inside the clump by using the following equation,

$$
M_{\text {clump }}=\mu_{\mathrm{H}_{2}} m_{\mathrm{H}} A_{\text {pixel }} \Sigma N\left(\mathrm{H}_{2}\right)
$$

where $\mathrm{m}_{\mathrm{H}}$ is the mass of hydrogen nucleus, $\mathrm{A}_{\text {pixel }}$ is the pixel area in $\mathrm{cm}^{2}, \mu_{\mathrm{H}_{2}}$ is the mean molecular weight and $\Sigma N\left(\mathrm{H}_{2}\right)$ is the integrated column density within the clump apertures. The clumps apertures are retrieved from the clumpfind algorithm. 

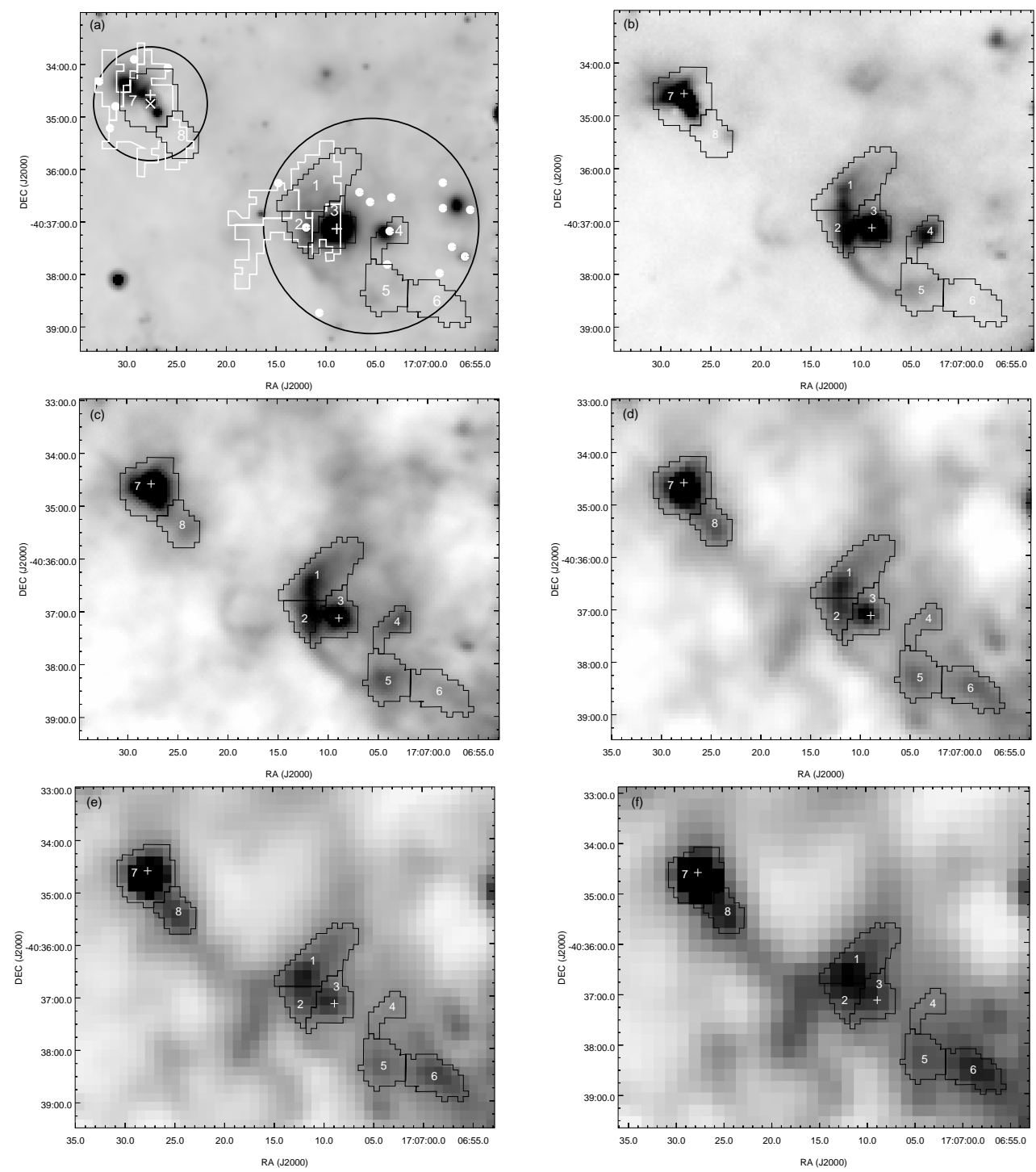

Fig. 9. - The eight clumps (apertures shown as black contours) detected from $250 \mu \mathrm{m}$ image are shown on (a) $24 \mu \mathrm{m}$ (b) PACS $70 \mu \mathrm{m}$ (c) PACS $160 \mu \mathrm{m}$ (d) SPIRE $250 \mu \mathrm{m}$ (e) SPIRE $350 \mu \mathrm{m}$ (f) SPIRE $500 \mu \mathrm{m}$ images. The retrieved clump apertures from the $1.2 \mathrm{~mm}$ map (Beltrán et al. 2006) are shown as white contours in (a) where the two regions are also marked as black circles. The ' + ' symbols mark the position of IRAS point sources associated with the regions. The positions of the identified YSOs (see Section 4.2) are shown on the $24 \mu \mathrm{m}$ image. 
2. From $250 \mu \mathrm{m}$ image: Here, the masses of the clumps are estimated from the $250 \mu \mathrm{m}$ integrated flux values obtained using the clumpfind algorithm and the following expression from Kauffmann et al. (2008)

$$
M=0.12 M_{\odot}\left(e^{1.439(\lambda / \mathrm{mm})^{-1}\left(T_{d} / 10 \mathrm{~K}\right)^{-1}}-1\right)\left(\frac{\kappa_{\nu}}{0.01 \mathrm{~cm}^{2} \mathrm{~g}^{-1}}\right)^{-1}\left(\frac{S_{\nu}}{\mathrm{Jy}_{\mathrm{y}}}\right)\left(\frac{D}{100 \mathrm{pc}}\right)^{2}\left(\frac{\lambda}{\mathrm{mm}}\right)^{3}
$$

where $T_{d}$ is the dust temperature, $\kappa_{\nu}$ is the dust opacity which is taken as $0.1\left(\frac{\nu}{1000 G H z}\right)^{\beta}$, D is the distance, $S_{\nu}$ is the integrated flux. For $T_{d}$, we use the mean dust temperatures of the clumps estimated from the temperature maps.

The derived masses and other physical properties of the clumps are listed in Table 4. As seen from the table, the masses derived from the column densities are lower (by an average factor of $\sim 0.9$ ) compared to those derived from the $250 \mu \mathrm{m}$ image alone. The masses derived from the column density map would be a better estimate given that it uses data from four bands. The table also lists the linear diameters of the clumps. We have estimated the deconvolved sizes following the method outlined in Beltrán et al. (2006). We also list the diameters derived based on the physical size of the clump (Kauffmann \& Pillai 2010) in parenthesis. The later does not have the beam effect removed and we refer to it as the effective diameter.

Table 4: Physical parameters of the clumps. $F_{250}$ is total flux density in $250 \mu \mathrm{m}$. The listed positions correspond to the peaks of the clumps as derived from the $250 \mu \mathrm{m}$ image using the clumpfind algorithm. The linear diameter listed here are the deconvolved (without parentheis) and the effective diameter (within parenthesis). $T_{d}$ and $N\left(\mathrm{H}_{2}\right)$ are the mean dust temperature and column density respectively. $\mathrm{M}_{250}$ is mass calculated using fluxes from $250 \mu \mathrm{m}$ and $\mathrm{M}_{\mathrm{CD}}$ is the mass calculated using the column density map.

\begin{tabular}{|c|c|c|c|c|c|c|c|c|c|}
\hline Clump No. & $\begin{array}{c}\text { RA (2000) } \\
\text { (hh:mm:ss.ss) }\end{array}$ & $\begin{array}{c}\text { DEC (2000) } \\
\text { (dd:mm:ss.ss) }\end{array}$ & $\begin{array}{l}F_{250} \\
(\mathrm{Jy})\end{array}$ & $\begin{array}{c}\text { Linear Diameter } \\
(\mathrm{pc})\end{array}$ & $\begin{array}{c}\text { Mean } T_{d} \\
(\mathrm{~K})\end{array}$ & $\begin{array}{l}\text { Mean } N\left(\mathrm{H}_{2}\right) \\
\left(\times 10^{22} \mathrm{~cm}^{-2}\right)\end{array}$ & $\begin{array}{l}\mathrm{M}_{250} \\
\left(\mathrm{M}_{\odot}\right)\end{array}$ & $\sum_{\left(\times 10^{23} \mathrm{~cm}^{-2}\right)}^{N\left(\mathrm{H}_{2}\right)}$ & $\begin{array}{l}\mathrm{M}_{\mathrm{CD}} \\
\left(\mathrm{M}_{\odot}\right)\end{array}$ \\
\hline \multicolumn{10}{|c|}{$\mathrm{S} 10$} \\
\hline 1 & $17: 07: 12.02$ & $-40: 36: 33.00$ & 222 & $1.1(1.9)$ & 20.6 & 2.0 & 1436 & 4.2 & 1390 \\
\hline 2 & $17: 07: 12.02$ & $-40: 36: 57.00$ & 85 & $0.2(1.1)$ & 20.8 & 1.7 & 533 & 1.1 & 354 \\
\hline 3 & $17: 07: 09.40$ & $-40: 37: 09.09$ & 131 & $0.6(1.4)$ & 21.5 & 1.7 & 750 & 2.1 & 685 \\
\hline 4 & $17: 07: 03.08$ & $-40: 37: 15.40$ & 63 & $0.3(1.0)$ & 21.0 & 1.6 & 390 & $\begin{array}{l}2.1 \\
1.0\end{array}$ & 337 \\
\hline 5 & $17: 07: 04.70$ & $-40: 38: 27.90$ & 134 & $0.6(1.5)$ & 20.5 & 1.8 & 875 & 2.5 & 845 \\
\hline 6 & $17: 06: 58.90$ & $-40: 38: 27.60$ & 143 & $0.7(1.6)$ & 19.6 & 2.1 & 1074 & 2.6 & 852 \\
\hline \multicolumn{10}{|c|}{ EGO345 } \\
\hline 7 & $17: 07: 27.77$ & $-40: 34: 44.05$ & 283 & $0.7(1.9)$ & 21.0 & 2.3 & 1754 & 4.7 & 1564 \\
\hline 8 & $17: 07: 24.63$ & $-40: 35: 26.24$ & 99 & $0.3(1.3)$ & 19.4 & 2.2 & 770 & 2.0 & 655 \\
\hline
\end{tabular}

In order to understand the nature of the sources towards these clumps, we use the online SED model fitting tool of Robitaille et al. (2007) to fit the clump fluxes with the inbuilt YSO models. This is along the lines discussed in Zavagno et al. (2010). Here, we assume that each clump would produce a single high-mass star. Apart from the MIPS and Herschel data, we have used $870 \mu \mathrm{m}$ ATLASGAL and $1.2 \mathrm{~mm}$ (Beltrán et al. 2006) fluxes. We use the clump apertures retrieved from the clumpfind algorithm to obtain flux densities in all wavelengths. Same apertures 
were used on nearby 'smooth' and 'dark' regions to estimate the background emission which is subtracted out from the clump fluxes. As done earlier, we take a conservative uncertainty of $15 \%$ on the background subtracted flux densities. Figure 10 shows the results of the fits towards the clumps. In Table 5, we list the range of values of various parameters of the first ten best fitting SED models with the best fit values in parenthesis. The envelope masses retrieved from fitting the SED models are seen to be $\sim 1.5-3$ times larger than the derived masses of the clumps except for Clump 4 where both the masses are similar. All clumps are seen to harbour high luminosity, high envelope accretion rate and massive YSOs. As mentioned in Section 4.3, the retrieved values of the parameters are to be used as indicative only as these models involve a large range in parameters with limited data points. Hence, instead of fitting to a unique combination, the models return a range in the parameter space.
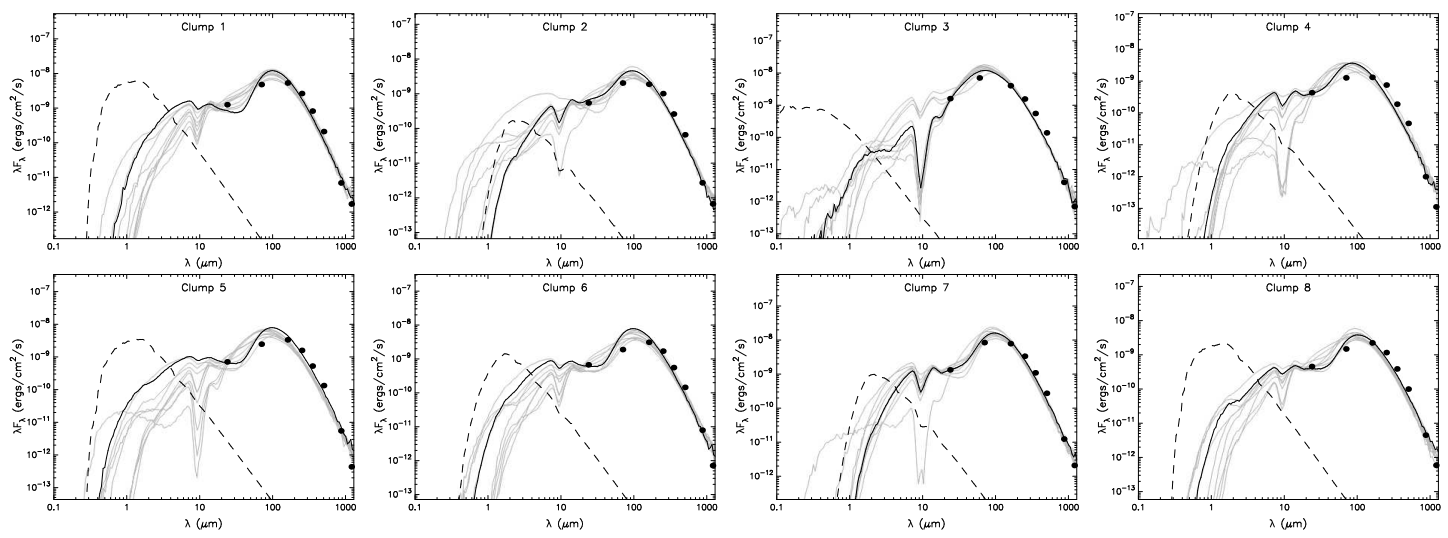

Fig. 10.- Results of the online SED modelling of Robitaille et al. (2007) for the eight clumps. The grey lines are the ten best fitting models and the black line is the best fit model.

Kauffmann \& Pillai (2010) suggest an empirical mass-radius relation to define a threshold for clouds to form massive stars. They derive this relation by comparing the mass-radius relation of clouds with and without massive star formation. The clouds devoid of massive star formation are shown to generally obey the relation, $m(r) \leqslant 870 \mathrm{M}_{\odot}(\mathrm{r} / \mathrm{pc})^{1.33}$. In Figure 11(a), we plot the estimated mass (from column density map) as a function of the effective radius of the clumps. It should be noted here that the threshold estimated by Kauffmann \& Pillai (2010) is based on effective radii derived using the physical area of the clumps. Hence, if we look at the filled circles in the figure, most of the clumps detected in the regions associated with S10 and EGO345 are above the threshold. Two clumps are seen just below but very close to the dividing line. This implies that all the clumps are potential high-mass star forming regions. We have also plotted the deconvolved radius of the clumps as open circles. The dashed line denotes the slope from Urquhart et al. (2013) and the region lying above that marks the location of high-mass star forming clumps. The relation given in Urquhart et al. (2013) is based on deconvolved sizes. Both these empirical mass-radius relations strongly suggest that the clumps detected in these regions are capable of forming high-mass 
Table 5: Physical properties derived from the ten best fitting SED models of Robitaille et al. (2007) for the eight detected clumps. The values in parenthesis are for the best fit models.

\begin{tabular}{ccccc}
\hline & & $\dot{M}_{\text {env }}$ & $M_{\text {env }}$ & Luminosity \\
Clump No. & $\left(\mathrm{M}_{\odot}\right)$ & $\left(10^{-3} \mathrm{M}_{\odot} \mathrm{yr}^{-1}\right)$ & $\left.\mathrm{M}_{\odot}\right)$ \\
\hline \multicolumn{5}{c}{$\mathrm{S} 10$} \\
\hline 1 & $12-22(19.7)$ & $5-9(9.2)$ & $2000-5000(2200)$ & $6-15(12.3)$ \\
2 & $9-14(10.8)$ & $2-7(5.0)$ & $400-2000(613)$ & $2-6(4.5)$ \\
3 & $11-22(11.7)$ & $2-9(2.3)$ & $1000-2000(1450)$ & $10-31(15.1)$ \\
4 & $8-12(11.8)$ & $1-5(3.3)$ & $100-700(333)$ & $2-9(4.1)$ \\
5 & $11-18(17.8)$ & $3-7(6.9)$ & $600-2500(1990)$ & $4-10(8.9)$ \\
6 & $12-18(17.8)$ & $4-7(6.9)$ & $2000-5000(1990)$ & $4-9(8.9)$ \\
\hline \multicolumn{5}{c}{ EGO345 } \\
\hline 7 & $15-25(24.8)$ & $5-10(9.3)$ & $2000-5000(4410)$ & $11-26(18.3)$ \\
8 & $10-14(11.5)$ & $2-6(5.1)$ & $600-3000(1820)$ & $2-6(4.4)$ \\
\hline
\end{tabular}

stars.

Clumps 3, 4, 7 show the presence of $24 \mu \mathrm{m}$ emission peaks of which Clumps 4 and 7 also include radio peaks. Clump 7 includes the EGO and Class I and II methanol masers. An intermediate mass YSO, IRS1 is shown to be located in Clump 4. Given these signatures of star formation, these three clumps can be considered to be active high-mass star forming clumps. Of these, Clump 3 seems to be in the earliest evolutionary phase prior to the formation of UCHII region. The peak of the bright radio emitting region lies in Clump 8. Apart from this, rest of the clumps do not reveal any signposts of active star formation. Following the discussion in Molinari et al. (2008) and Giannetti et al. (2013), these could be regarded as either being starless or with a deeply embedded ZAMS star. All clumps in our sample have luminosities, $L>10^{3} L_{\odot}$ and hence are likely to host ZAMS stars (Giannetti et al. 2013).

To further understand the evolutionary phase of these clumps, we follow the discussion in Molinari et al. (2008) which is based on the SED of massive YSOs. They discuss the evolutionary sequence of massive YSOs on a $\mathrm{L}_{b o l}-\mathrm{M}_{\text {env }}$ plot (see their Fig. 9). Their plot also includes the regime of low-mass YSOs from Saraceno et al. (1996) and shows the behaviour of the bolometric luminosity, $\mathrm{L}_{\mathrm{bol}}$ and the envelope mass, $\mathrm{M}_{\mathrm{env}}$ as the YSO moves from the accelerating accretion phase to the end of it reaching the ZAMS (or close to it) and then proceeding to the envelope clean-up phase. In Figure 11(b), we plot the clump masses (and the corresponding envelope masses determined from the SED models) as a function of the derived luminosities. The loci demarcating the accelerating accretion and onset of envelope clearing phases, adopted from Fig. 9 of Molinari et al. (2008), are also plotted in this figure. Our plot shows the high-mass end of their figure. Two of the active clumps (4 and 7 ) which show radio peaks are possibly in the early envelope clearing phase. This 

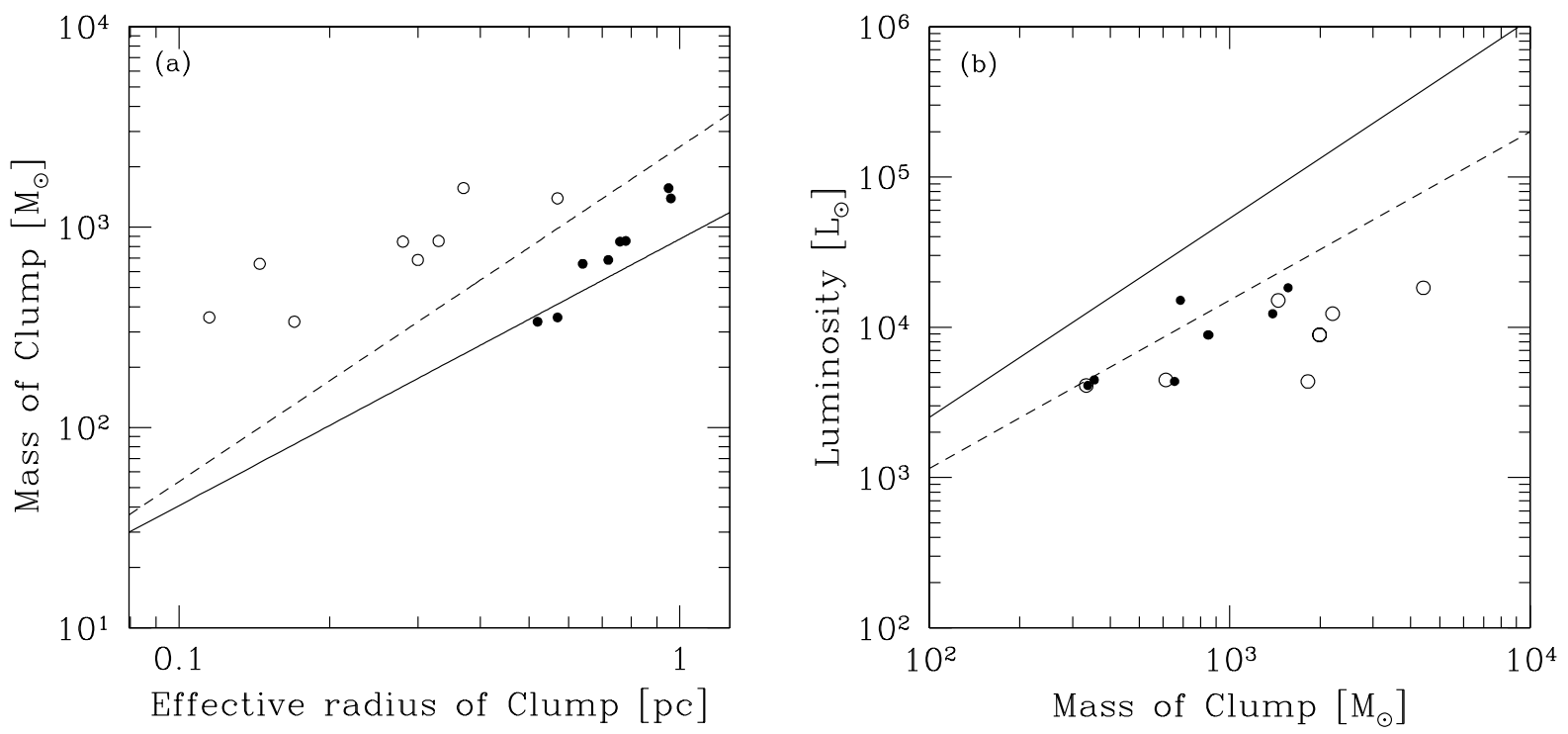

Fig. 11. - (a) Clump masses as a function of the effective radius. Filled circles denote the effective radii derived based on the physical sizes of the clumps and open circles are the deconvolved sizes (see text for details). The straight solid line shows the threshold for high-mass star formation based on the relation from Kauffmann \& Pillai (2010). Also plotted as the dashed line is the slope from Urquhart et al. (2013). (b) Clump luminosity as a function of mass. The solid and dashed lines are adopted from Fig. 9 of Molinari et al. (2008). These lines distinguish the accelerating accretion phase and the onset of envelope clearing phase. Filled circles represent the luminosity of the clumps as a function of the derived clump masses and the open circles represent the luminosity as a function of envelope masses derived from the SED modelling.

is consistent with the fact the ZAMS phase is marked by detectable ionized emission. Apart from clump 2, which is also close to the demarcating loci, rest of the clumps lie in the region associated with accelerating accretion phase of evolution. As discussed by these authors, the end of the ascending phase is accompanied by very high accretion rates which is consistent with the values obtained from the SED modelling of the clumps.

Figure 12(a) plots the envelope mass as a function of the final mass of the star, $M_{*}$ based on the best fit SED model values. The derived envelope mass can be considered here as the initial mass of the envelope given the almost vertical evolutionary track in the $L_{b o l}-M_{\text {env }}$ plot of Molinari et al. (2008) where the mass of the envelope remain the same from the initial to the end of accelerating accretion phase. As seen from the figure, the final mass of the star follows a decreasing trend with decrease in mass of the envelope. The figure also shows log-log fit from Molinari et al. (2008). The general trend and slope seen in our clumps are consistent with the fit adopted from the above paper but shifted to the left. Based on SED model fitted values, our results also indicate a disagreement 
with the prediction of competitive accretion model of Bonnell et al. (2004), where the final mass of a star is shown to be unrelated to the initial mass of the clump. From the estimated mass of the stars and the envelopes, we calculate the star formation efficiency, $\epsilon=\left[\frac{M_{*}}{M_{\text {env }}} \times 100\right]$ for the eight clumps. Figure 12(b) shows this as a function of the envelope mass. As is clearly evident from the plot, the efficiency decreases from $3.5 \%$ to $0.6 \%$ with increasing envelope mass which in this case is assumed to be the initial envelope mass. These are a factor of 2 on the lower side compared to the results obtained in Molinari et al. (2008) and the average star forming efficiency across the Galaxy as discussed in Williams \& McKee (1997). As discussed in Molinari et al. (2008), the estimated values of the star forming efficiency should be taken as a lower limit considering the fact that massive YSOs form in clusters alongwith low and intermediate-mass stars. The larger the clump mass, the more populous would be the cluster and hence relatively less mass goes to the most massive member. Hence, using the mass of the most massive member understates the star forming efficiencies.
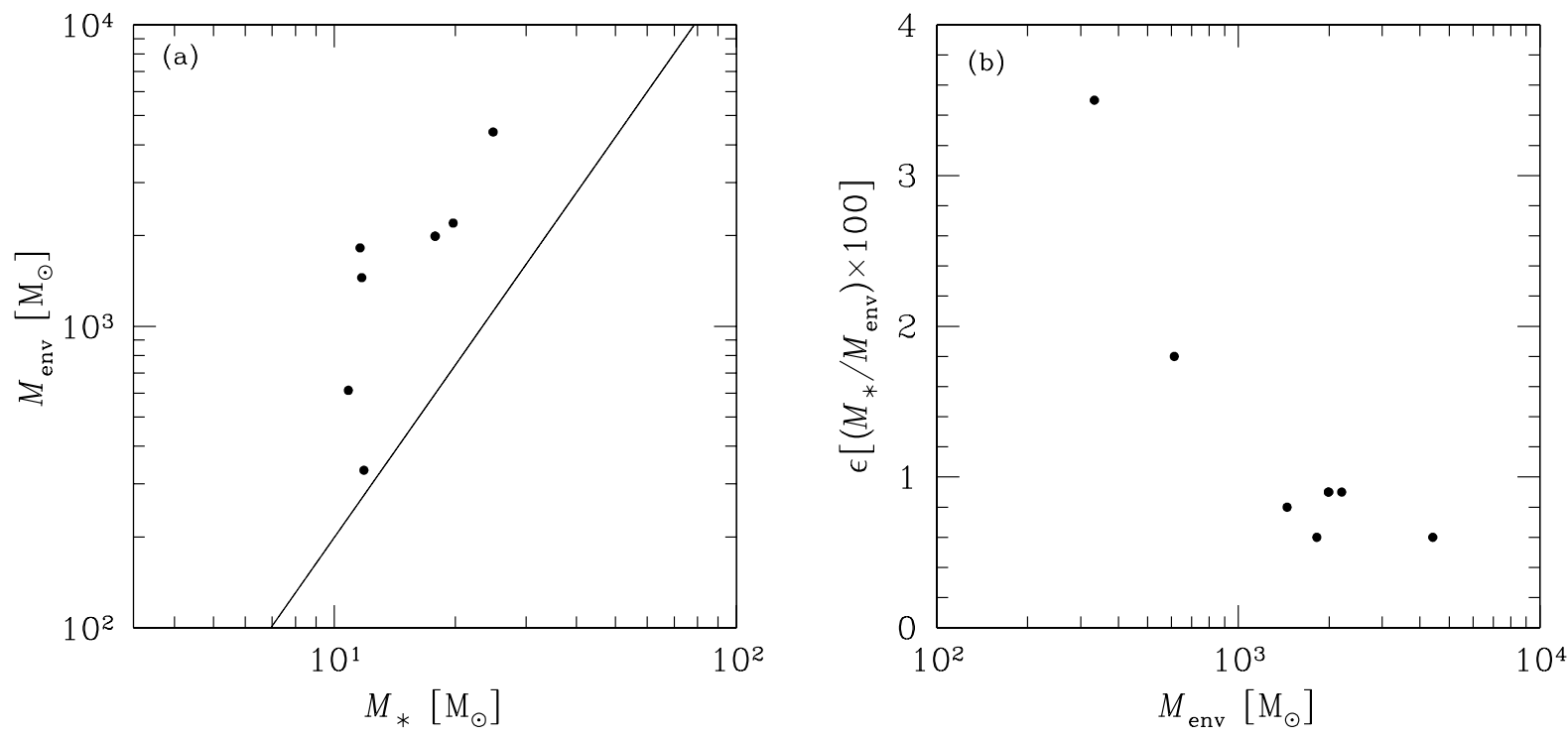

Fig. 12. - (a) The final mass of the massive star, $M_{*}$ as a function of the envelope mass (assumed to be the initial mass of the envelope here). The straight line is the fit adopted from Molinari et al. (2008). (b) The star forming efficiency of the clumps as a function of the envelope mass.

\subsection{Possible bow-wave in S10?}

Detailed study on the formation and nature of bubbles have been in focus since the first published catalogs of Churchwell et al. (2006, 2007) based on the Spitzer - GLIMPSE and MIPSGAL survey images. The observed bright-rimmed morphology in the MIR is a combination of UV radia- 
tion excited polycyclic aromatic hydrocarbons (PAHs) emission in the IRAC bands and the thermal emission from hot dust surrounding the newly formed star. Given the prominent MIR morphology, these are more commonly known as IR bubbles. The general bubble structure is a photodissociation region (PDR) visible at 5.8 and $8 \mu \mathrm{m}$ and an evacuated cavity within this (Churchwell et al. 2006, 2007; Watson et al. 2008, 2009; Anderson et al. 2010; Zavagno et al. 2010; Deharveng et al. 2010; Kendrew et al. 2012). More recently, another catalog of IR bubbles was published by Simpson et al. (2012) - The Milky Way Project.

As mentioned in the introduction, several feedback mechanisms are believed to be responsible for the formation of the bubbles. Even though the relevance of each depends on the nature of the ionizing star, the traditional picture of wind-blown bubbles (Weaver et al. 1977) lacks observational support as outlined in Ochsendorf et al. (2014a). Non-detection of X-ray emission inside bubbles and presence of dust in the HII regions are observations which challenge the wind blown bubble model. The view that evaporation of dense cloudlets replenishes the interior of bubbles with new generation of dust grains could explain the presence of dust seen in the HII regions associated with the bubbles (Everett \& Churchwell 2010). This mechanism however fails to account for the growing evidence of arc-type structures seen at $24 \mu \mathrm{m}$ in the interior of bubbles and the observation of incomplete shells in HII bubbles (Watson et al. 2008; Kang et al. 2009; Deharveng et al. 2010).

Ochsendorf et al. (2014a, b) have explored the formation of infrared bubbles for weak wind stars $\left(\log \left(\mathrm{L} / \mathrm{L}_{\odot}\right) \lesssim 5\right)$ which invokes thermal pressure of the ionized gas instead of stellar wind. The two dimensional hydrodynamical simulations of this model by Ochsendorf et al. (2014b) focuses on the formation of arc-type structures seen to exist close to the ionizing star in the bubble interior. Refering to Fig. 2 of Ochsendorf et al. (2014b), the newly born massive star starts of with ionizing the surrounding and forming an expanding sphere of ionized gas. The thermal pressure in the interior causes the bubble to expand sweeping up neutral gas in a dense encompassing shell. Formation of a shock front may occur provided the expansion is supersonic. In case of a density gradient or a break in the bubble shell, the ionized gas is shown to flow towards the low density regions and leaks out to the surrounding ISM. This releases the pressure of the overpressurized bubble. Along with the ionized gas the dust is also dragged along but is halted in the flow direction by the radiation pressure forming a dust or bow wave which show up as arcs in the mid-infrared wavelengths. This model simulation finds observational validation in the arcs seen at $24 \mu \mathrm{m}$ around $\sigma$ Ori AB (Ochsendorf et al. 2014a) which is possibly the first detection of the predicted radiation driven dust wave around a weak wind star. Similar arcs detected in the interiors of bubbles RCW 120 and RCW 82 are also well explained by this model (Ochsendorf et al. 2014b).

The scenario associated with bubble S10 is rather interesting. In Figure 13, we show the three colour composite image of S10 using $8 \mu \mathrm{m}$ (Spitzer - IRAC), $24 \mu \mathrm{m}$ (Spitzer - MIPS) and $610 \mathrm{MHz}$ (GMRT). $8 \mu \mathrm{m}$ emission is seen as a prominent outer shell and an inner arc-type feature as mentioned earlier. $24 \mu \mathrm{m}$ emission shows enhanced distribution mostly in three localized regions. These are (1) near the eastern limb of the outer shell coincident with the position of the IRAS point source, (2) toward the centre of the bubble with the inner $8 \mu \mathrm{m}$ arc enveloping it and (3) beyond the 
periphery of the broken western part of the bubble. The $8 \mu \mathrm{m}$ emission shows a rupture in the outer shell which seems to be aligned (at a PA of $\sim 50^{\circ}$ north of east) to the opening direction of the inner arc-type feature as is seen clearly in the right panel of Figure 13. The arc-type inner structure and the ruptured outer shell morphology is also clearly seen at $5.8 \mu \mathrm{m}$. The ionized emission at $610 \mathrm{MHz}$ displays a fan-like morphology aligned in this direction. Though less extended, the $1280 \mathrm{MHz}$ map also reveals similar structure (see Figure 3). The radio emission displays a picture wherein a flow of ionized gas is seen from the position of the radio peak (considered to be the position of the ionizing star) towards lower density regions and further leaking out of the rupture in the outer shell. This interesting morphology prompted us to investigate the presence of a bow-wave but at shorter wavelengths compared to the $24 \mu \mathrm{m}$ arcs discussed in Ochsendorf et al. (2014a, b $)$. This is supported by the fact that the likely ionizing star responsible for S10 falls in the 'weak-wind' category with an estimated $\log \left(\mathrm{L} / \mathrm{L}_{\odot}\right)$ lying between $4.04(\mathrm{~B} 0.5)$ and $4.40(\mathrm{~B} 0)$.

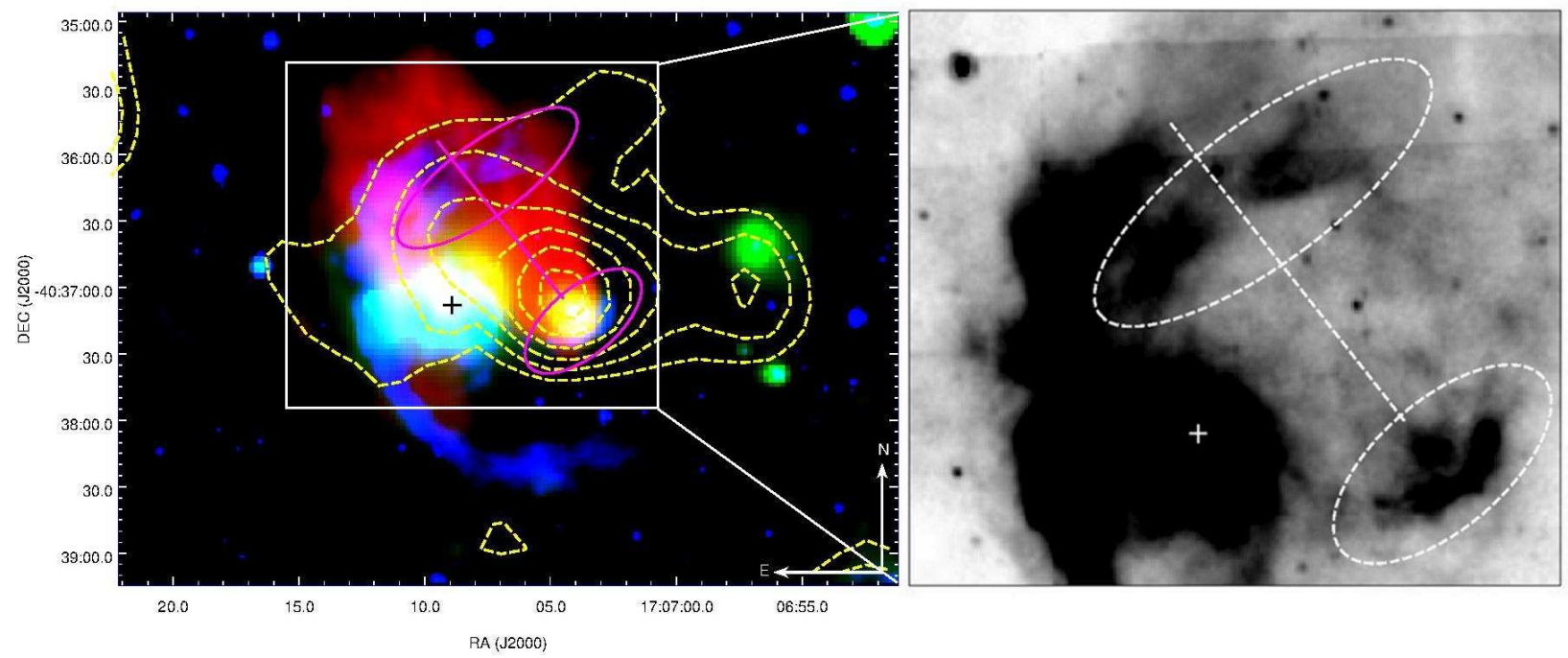

Fig. 13. - Left panel: Three-color composite image of the region associated with the bubble S10 with $8 \mu \mathrm{m}$ Spitzer-GLIMPSE (blue), $24 \mu \mathrm{m}$ MIPSGAL (green), and $610 \mathrm{MHz}$ GMRT (red). Low resolution radio emission at $843 \mathrm{MHz}$ from SUMSS is shown as contours. Right panel: Enlarged view of the outer shell rupture and the arc-type feature in the $8 \mu \mathrm{m}$ image.

We assume the expansion of the HII region around the massive B0.5 - B0 star (located at the radio peak) to be responsible for the formation of the bubble that is seen as the outer (and larger) shell. This implies that the $8 \mu \mathrm{m}$ band emission seen in this outer shell is largely due to PAH emission in the PDR with contribution from thermal emission from dust as well (Watson et al. 2008; Pomarès et al. 2009). It is well known that intense UV radiation close to the ionizing star destroys the PAH molecules (Watson et al. 2008). Hence, the $8 \mu \mathrm{m}$ inner arc-type feature close to the possible ionizing star is likely to be due to thermal emission from dust alone. As seen in the figure, a bright $24 \mu \mathrm{m}$ blob overlaps the radio emission towards centre of the bubble. $24 \mu \mathrm{m}$ 
emission arises mostly near the hot star when the dust is heated to $\sim 100 \mathrm{~K}$. The $8 \mu \mathrm{m}$ arc-type emission is also seen to be coupled to the ionized gas. As discussed in Ochsendorf et al. (2014b), the gas and dust coupling depends on the efficiency of momentum transfer between gas and dust which would result in either a dust-wave (gas and dust decoupled) or a bow-wave (gas and dust spatially correlated). The gas and dust couple well in relatively slower flow of ionized gas. The bow-wave is similar in appearance to the stellar-wind bow-shock (van Buren et al. 1990). However, in case of the bow-wave, the dust grains are stalled at a distance $\left(r_{\min }\right)$ exceeding the stand-off distance $\left(r_{s}\right)$ of the bow-shock in the flow direction (Ochsendorf et al. 2014a).

The stand-off distance, $r_{s}$, is determined using the following expressions based on Mac Low et al. (1991) which equates the momentum flux of the stellar wind with the ram pressure of the star moving through the ISM.

$$
\begin{gathered}
r_{s}=1.78 \times 10^{3} \sqrt{\frac{\dot{M} v_{w}}{\mu_{\mathrm{H}} n_{\mathrm{H}} v_{\star-\mathrm{ISM}}^{2}}} \mathrm{pc} \\
\dot{m}=2.0 \times 10^{-7}\left(L / L_{\odot}\right)^{1.25} \\
\log v_{w}^{\prime}=-38.2+16.23 \log T_{\mathrm{eff}}-1.70\left(\log T_{\mathrm{eff}}\right)^{2}
\end{gathered}
$$

where, $\dot{M}\left(=\dot{m} \times 10^{-6} \mathrm{M}_{\odot} \mathrm{yr}^{-1}\right)$ is the mass-loss rate from the star and $v_{w}\left(=v_{w}^{\prime} \times 10^{3} \mathrm{~km} \mathrm{~s}^{-1}\right)$ is the terminal velocity of the stellar wind, $\mu_{\mathrm{H}}$ is the mean mass per hydrogen nucleus, $n_{\mathrm{H}}$ is the hydrogen gas density in $\mathrm{cm}^{-3}, v_{\star-I S M}$ is the velocity of star with respect to the ISM in $\mathrm{km} \mathrm{s}^{-1}$, $L$ is the stellar luminosity, $L_{\odot}$ is the solar luminosity and $T_{\text {eff }}$ is the effective temperature of star, respectively. The hydrogen gas density $n_{\mathrm{H}}$ is determined from the column density maps obtained using the Herschel images (see Section 4.4.1). Assuming uniform density in a spherical region within $\sim 15^{\prime \prime}$ of the peak of radio emission (position of the ionizing source), we estimate $n_{\mathrm{H}}$ to be $1.6 \times 10^{4} \mathrm{~cm}^{-3}$. This is of the same order obtained for the clumps by Beltrán et al. (2006). Taking $\mu_{\mathrm{H}}=1.4$ and assuming a typical velocity, $v_{\star-I S M}$ of $10 \mathrm{~km} \mathrm{~s}^{-1}$, we get a stand-off distance between $0.8-1.5 \times 10^{-2} \mathrm{pc}$ which corresponds to $0.3^{\prime \prime}-0.5^{\prime \prime}$ at a distance of $5.7 \mathrm{kpc}$ for spectral type of B0.5 - B0 estimated for the ionizing star. The values for $L$ and $T_{\text {eff }}$ are taken from Panagia (1973). From the 5.8 and $8 \mu \mathrm{m}$ images we estimate the arc to be at a distance $\left(r_{\min }\right)$ of $\sim 15^{\prime \prime}$ from the radio peak which corresponds to $\sim 0.4 \mathrm{pc}$, far exceeding the stand-off distance, $r_{s}$. This is consistent with what is expected for a bow-wave to occur. In Ochsendorf et al. (2014a), a similar dust structure qualifying as a dust-wave is seen at a distance of $0.1 \mathrm{pc}$ from $\sigma$ Orionis AB. Further, Fig. 13 of Ochsendorf et al. (2014a) shows $r_{s}$ and $r_{\text {min }}$ as a function of the ISM density for the strong and weak wind regimes and clearly shows that the formation of dust and bow-waves are more efficient around weak-wind stars. The ratio $r_{\text {min }} / r_{s}$ roughly estimated from the figure for the $n_{\mathrm{H}}$ value of $\mathrm{S} 10\left(1.6 \times 10^{4} \mathrm{~cm}^{-3}\right)$ is around 45 . This is fairly consistent with the range $\sim 25-50$ obtained in our case. 
Driven by the radio and MIR morphology and based on the above calculations, we propose that the inner arc-type structure seen in the mid-infrared bands at 5.8 and $8 \mu \mathrm{m}$ surrounding the weak-wind ionizing star is a radiation-pressure driven dust structure: a bow-wave. The radiation pressure of the ionizing star of S10 stops the dust that is being dragged along the flow of the ionized gas at a distance that exceeds the stand-off distance. This inference is further supported by the radio maps at 610,1280 , and $843 \mathrm{MHz}$ which associates the ionized emission with the bubble that is traced by the outer shell. In addition, the orientation of the inner arc and the rupture on the outer shell and the proximity of the inner arc to the ionizing star mostly excludes the possibility of the inner arc being part of a different bubble. However, our simplistic arguments in favour of the bow-wave fail to convincingly explain the following aspects - (1) the bow-wave is not revealed in the $24 \mu \mathrm{m}$ emission which appears almost spherical in our case; (2) an incomplete or broken morphology is seen toward the western part of the bubble. If we assume the bubble formation to proceed as proposed by Ochsendorf et al. (2014a , b) , then the western limb also needs to be blown out by the flow of ionized gas in that direction. Hence, one would expect the ionized gas morphology to be consistent with the above. Our results do not clearly show this. However it should be noted that the lowest $1280 \mathrm{MHz}$ contour (see Fig. 3) shows a small protrusion and the low resolution $843 \mathrm{MHz}$ radio emission shows an extension in the direction of the broken western part of the bubble; (3) it is not clear why the likely bow-wave is seen only towards the narrower rupture and no such feature is in the eastern side facing the larger western opening. It is however possible that the direction of the flow of ionized gas and hence the dust drag is dictated by the local density gradient close to the ionizing source. The aforementioned discrepancies suggest that a detailed study of the dust grain characteristics and its wavelength dependence is necessary before we can address conclusively the possibility of occurrence of a bow-wave at shorter MIR wavelengths.

\section{Summary}

In this paper we have done a multiwavelength study towards southern infrared bubble S10. We probed two regions S10 and EGO345 and arrive at the following conclusions.

1. The radio maps at 610 and $1280 \mathrm{MHz}$ show the presence of ionized emission in the interior of the bubble with the emission being more extended at $610 \mathrm{MHz}$. A steep density gradient is also evident from the $610 \mathrm{MHz}$ emission which increases towards the likely centre of the bubble. Assuming optically thin, free-free emission from a single ionizing star, the spectral type of it is determined to be B0.5 - B0. The region associated with EGO345 also show the presence of ionized emission at both the above radio frequencies. The morphology is compact and nearly spherical at $610 \mathrm{MHz}$ compared to a relatively clumpier and extended one at $1280 \mathrm{MHz}$. The spectral type of the ionizing source responsible for this emission is estimated to be B0 - O9.5.

2. An intermediate-mass YSO of Class I/II, IRS1, with estimated mass of $6.2 \mathrm{M}_{\odot}$ lies $\sim 7^{\prime \prime}$ to 
the west of the radio peak of S10. It is unlikely that this is the NIR counterpart of the ionizing star. The massive star responsible for the ionized region could likely be a deeply embedded source.

3. Dust temperature and column density maps are generated using SED modelling of the thermal dust emission from Herschel FIR data. The distribution of ionized gas traced by the radio emission is found to be consistent with location of warmer dust. The column density map reveals the presence of several high density clumps and filaments.

4. Using the $250 \mu \mathrm{m}$ image and the $2 \mathrm{D}$ variation of the clumpfind algorithm, eight clumps are detected in both the regions. The masses of clumps as derived from the column density maps range between $\sim 337-1564 \mathrm{M}_{\odot}$. The mass and effective radii of the clumps place them in the high-mass star-forming clumps regime. Clumps \# 3, 4, and 7 show signatures of active star formation with Clumps \# 4 and 7 coincident with the radio peaks of S10 and EGO345, respectively.

5. SED modelling for sources towards these clumps show that they harbour high-luminosity, high envelope accretion rate, massive YSOs. Based on the fitted values of mass of star and envelope, these clumps are seen to lie in the accelerating accretion phase of massive YSOs.

6. The MIR images show the presence of an arc-like feature near the likely centre of the bubble aligned with a rupture seen in the outer shell of the bubble. The arc encompasses the radio emission on the south-west side. The ionized emission at both the radio frequencies is consistent with the picture of a flow of ionized gas towards the outer shell originating from the centre of the bubble. The above scenario indicates at a possible detection of a bow-wave at the MIR wavelengths. This is supported by the stand-off distance which is estimated to be much smaller than the distance of the arc from the radio peak as is the case with bow-waves.

Acknowledgment: We thank the referee for his/her valuable comments and suggestions which has helped improve the quality of the paper. We thank the staff of the GMRT, that made the radio observations possible. GMRT is run by the National Centre for Radio Astrophysics of the Tata Institute of Fundamental Research. We thank R. Cesaroni for providing the $1.2 \mathrm{~mm}$ maps of the regions. We thank Varsha Ramachandran for help in Python programming.

\section{REFERENCES}

Allen, L. E., Calvet, N., D'Alessio, P., et al. 2004, ApJS, 154, 363

Anderson, L. D., Zavagno, A., Rodón, J. A., et al. 2010, A\&A, 518, L99

André, P., Men'shchikov, A., Bontemps, S., et al. 2010, A\&A, 518, L102

Arthur, S. J., Kurtz, S. E., Franco, J., \& Albarrán, M. Y. 2004, ApJ, 608, 282 
Battersby, C., Bally, J., Ginsburg, A., et al. 2011, A\&A, 535, A128

Beckwith, S. V. W., Sargent, A. I., Chini, R. S., \& Guesten, R. 1990, AJ, 99, 924

Beltrán, M. T., Brand, J., Cesaroni, R., et al. 2006, A\&A, 447, 221

Benjamin, R. A., Churchwell, E., Babler, B. L., et al. 2003, PASP, 115, 953

Bonnell, I. A., Vine, S. G., \& Bate, M. R. 2004, MNRAS, 349, 735

Caratti o Garatti, A., Stecklum, B., Linz, H., Garcia Lopez, R., \& Sanna, A. 2015, A\&A, 573, A82

Caswell, J. L., Fuller, G. A., Green, J. A., et al. 2010, MNRAS, 404, 1029

Chambers, E. T., Jackson, J. M., Rathborne, J. M., \& Simon, R. 2009, ApJS, 181, 360

Chen, X., Ellingsen, S. P., Shen, Z.-Q., Titmarsh, A., \& Gan, C.-G. 2011, ApJS, 196, 9

Churchwell, E. 2002, ARA\&A, 40, 27

Churchwell, E., Povich, M. S., Allen, D., et al. 2006, ApJ, 649, 759

Churchwell, E., Watson, D. F., Povich, M. S., et al. 2007, ApJ, 670, 428

Cyganowski, C. J., Whitney, B. A., Holden, E., et al. 2008, AJ, 136, 2391

De Buizer, J. M., \& Vacca, W. D. 2010, AJ, 140, 196

Deharveng, L., Peña, M., Caplan, J., \& Costero, R. 2000, MNRAS, 311, 329

Deharveng, L., Schuller, F., Anderson, L. D., et al. 2010, A\&A, 523, A6

Everett, J., \& Churchwell, E. 2010, in Astronomical Society of the Pacific Conference Series, Vol. 438, The Dynamic Interstellar Medium: A Celebration of the Canadian Galactic Plane Survey, ed. R. Kothes, T. L. Landecker, \& A. G. Willis, 69

Faimali, A., Thompson, M. A., Hindson, L., et al. 2012, MNRAS, 426, 402

Fontani, F., Beltrán, M. T., Brand, J., et al. 2005, A\&A, 432, 921

Galametz, M., Kennicutt, R. C., Albrecht, M., et al. 2012, MNRAS, 425, 763

Giannetti, A., Brand, J., Sánchez-Monge, Á., et al. 2013, A\&A, 556, A16

Griffin, M. J., Abergel, A., Abreu, A., et al. 2010, A\&A, 518, L3

Gutermuth, R. A., Myers, P. C., Megeath, S. T., et al. 2008, ApJ, 674, 336

Haslam, C. G. T., Salter, C. J., Stoffel, H., \& Wilson, W. E. 1982, A\&AS, 47, 1 
Hildebrand, R. H. 1983, QJRAS, 24, 267

Inoue, A. K., Hirashita, H., \& Kamaya, H. 2001, ApJ, 555, 613

Ji, W.-G., Zhou, J.-J., Esimbek, J., et al. 2012, A\&A, 544, A39

Kang, M., Bieging, J. H., Kulesa, C. A., \& Lee, Y. 2009, ApJ, 701, 454

Kauffmann, J., Bertoldi, F., Bourke, T. L., Evans, II, N. J., \& Lee, C. W. 2008, A\&A, 487, 993

Kauffmann, J., \& Pillai, T. 2010, ApJ, 723, L7

Kendrew, S., Simpson, R., Bressert, E., et al. 2012, ApJ, 755, 71

Launhardt, R., Stutz, A. M., Schmiedeke, A., et al. 2013, A\&A, 551, A98

Lee, H.-T., Takami, M., Duan, H.-Y., et al. 2012, ApJS, 200, 2

Lee, H.-T., Liao, W.-T., Froebrich, D., et al. 2013, ApJS, 208, 23

Liu, H.-L., Li, J.-Z., Wu, Y., et al. 2016, ApJ, 818, 95

Mac Low, M.-M., van Buren, D., Wood, D. O. S., \& Churchwell, E. 1991, ApJ, 369, 395

Mallick, K. K., Ojha, D. K., Tamura, M., et al. 2015, MNRAS, 447, 2307

Marcote, B., Ribó, M., Paredes, J. M., \& Ishwara-Chandra, C. H. 2015, MNRAS, 451, 59

Mauch, T., Murphy, T., Buttery, H. J., et al. 2003, MNRAS, 342, 1117

McKee, C. F., \& Tan, J. C. 2003, ApJ, 585, 850

Mezger, P. G., \& Henderson, A. P. 1967, ApJ, 147, 471

Molinari, S., Pezzuto, S., Cesaroni, R., et al. 2008, A\&A, 481, 345

Molinari, S., Swinyard, B., Bally, J., et al. 2010, A\&A, 518, L100

Ochsendorf, B. B., Cox, N. L. J., Krijt, S., et al. 2014a, A\&A, 563, A65

Ochsendorf, B. B., Verdolini, S., Cox, N. L. J., et al. 2014b, A\&A, 566, A75

Panagia, N. 1973, AJ, 78, 929

Paron, S., Petriella, A., \& Ortega, M. E. 2011, A\&A, 525, A132

Pitann, J., Linz, H., Ragan, S., et al. 2013, ApJ, 766, 68

Poglitsch, A., Waelkens, C., Geis, N., et al. 2010, A\&A, 518, L2

Pomarès, M., Zavagno, A., Deharveng, L., et al. 2009, A\&A, 494, 987 
Rieke, G. H., Young, E. T., Engelbracht, C. W., et al. 2004, ApJS, 154, 25

Robitaille, T. P., Whitney, B. A., Indebetouw, R., \& Wood, K. 2007, ApJS, 169, 328

Robitaille, T. P., Meade, M. R., Babler, B. L., et al. 2008, AJ, 136, 2413

Roger, R. S., Costain, C. H., Landecker, T. L., \& Swerdlyk, C. M. 1999, A\&AS, 137, 7

Saraceno, P., Andre, P., Ceccarelli, C., Griffin, M., \& Molinari, S. 1996, A\&A, 309, 827

Schraml, J., \& Mezger, P. G. 1969, ApJ, 156, 269

Simon, J. D., Bolatto, A. D., Whitney, B. A., et al. 2007, ApJ, 669, 327

Simpson, R. J., Povich, M. S., Kendrew, S., et al. 2012, MNRAS, 424, 2442

Swarup, G., Ananthakrishnan, S., Kapahi, V. K., et al. 1991, Current Science, Vol. 60, NO.2/JAN25, P. 95, 1991, 60, 95

Tan, J. C., Beltrán, M. T., Caselli, P., et al. 2014, Protostars and Planets VI, 149

Urquhart, J. S., Thompson, M. A., Moore, T. J. T., et al. 2013, MNRAS, 435, 400

van Buren, D., Mac Low, M.-M., Wood, D. O. S., \& Churchwell, E. 1990, ApJ, 353, 570

Vig, S., Ghosh, S. K., Ojha, D. K., \& Verma, R. P. 2007, A\&A, 463, 175

Ward-Thompson, D., \& Robson, E. I. 1990, MNRAS, 244, 458

Watson, C., Corn, T., Churchwell, E. B., et al. 2009, ApJ, 694, 546

Watson, C., Povich, M. S., Churchwell, E. B., et al. 2008, ApJ, 681, 1341

Weaver, R., McCray, R., Castor, J., Shapiro, P., \& Moore, R. 1977, ApJ, 218, 377

Williams, J. P., de Geus, E. J., \& Blitz, L. 1994, ApJ, 428, 693

Williams, J. P., \& McKee, C. F. 1997, ApJ, 476, 166

Wood, D. O. S., \& Churchwell, E. 1989, ApJS, 69, 831

Xue, X. X., Rix, H. W., Zhao, G., et al. 2008, ApJ, 684, 1143

Yorke, H. W., \& Sonnhalter, C. 2002, ApJ, 569, 846

Zavagno, A., Anderson, L. D., Russeil, D., et al. 2010, A\&A, 518, L101 